\title{
The Toxicology Investigators Consortium Case Registry—the 2017 Annual Report
}

\author{
Lynn A. Farrugia ${ }^{1}$ (1) - Sean H. Rhyee ${ }^{2}$ Sharan L. Campleman ${ }^{3} \cdot$ Bryan Judge $^{4} \cdot$ Louise Kao $^{5} \cdot$ Anthony Pizon $^{6}$.

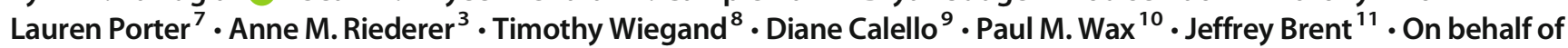 \\ the Toxicology Investigators Consortium (ToxIC) Study Group
}

Received: 12 July 2018 / Revised: 19 July 2018 / Accepted: 20 July 2018 / Published online: 9 August 2018

(C) American College of Medical Toxicology 2018

\begin{abstract}
The Toxicology Investigators Consortium (ToxIC) Case Registry was established by the American College of Medical Toxicology in 2010. The Registry collects data from participating sites with the agreement that all bedside medical toxicology consultations will be entered. The objective of this eighth annual report is to summarize the Registry's 2017 data and activity with its additional 7577 cases. Cases were identified for inclusion in this report by a query of the ToxIC database for any case entered from 1 January to 31 December 2017. Detailed data was collected from these cases and aggregated to provide information which includes demographics (e.g., age, gender, race, ethnicity), reason for medical toxicology evaluation (e.g., intentional pharmaceutical exposure, envenomation, withdrawal from a substance), agent and agent class, clinical signs and symptoms (e.g., vital sign abnormalities, organ system dysfunction), treatments and antidotes administered, fatality, and life support withdrawal data. Females were involved in 50.4\% of cases. Transgender demographic information collection was initiated in 2017 to better represent the population and there were 36 cases involving transgender patients. Adults aged 19-65 were the most commonly reported age group. Non-opioid analgesics were the most commonly reported agent class, with acetaminophen again the most common agent reported. There were 93 fatalities reported in 2017. Treatment interventions were frequently reported with $30.6 \%$ receiving specific antidotal therapy. Major trends in demographics and exposure characteristics remained similar to past years' reports. While treatment interventions were commonly required, fatalities were rare.
\end{abstract}

Keywords Poisonings $\cdot$ Overdose $\cdot$ Surveillance $\cdot$ Epidemiology $\cdot$ Medical toxicology

Electronic supplementary material The online version of this article (https://doi.org/10.1007/s13181-018-0679-z) contains supplementary material, which is available to authorized users.

Lynn A. Farrugia

Lynn.Farrugia@hhchealth.org

1 Hartford Hospital and University of Connecticut School of Medicine, 80 Seymour Street, Hartford, CT 06102, USA

2 University of Massachusetts Medical School, 55 Lake Avenue North, LA-202, Worcester, MA 01655, USA

3 American College of Medical Toxicolog, 10645 N. Tatum Blvd., Suite 200-111, Phoenix, AZ 85028, USA

4 Spectrum Health Blodgett Hospital, 1840 Wealthy Street SE, Grand Rapids, MI 49506, USA

5 Indiana University School of Medicine, 340 West 10th Street, Suite 6200, Indianapolis, IN 46202, USA
6 University of Pittsburgh School of Medicine and University of Pittsburgh Medical Center, 200 Lothrop Street, Pittsburgh, PA 15213, USA

7 Banner-University Medical Center Phoenix, 925 E. McDowell Rd, Phoenix, AZ 85006, USA

8 University of Rochester Medical Center and Strong Memorial Hospital, 601 Elmwood Ave, Rochester, NY 14642, USA

9 New Jersey Medical School, Rutgers, The State University of New Jersey, 140 Bergen Street, Suite G1600, Newark, NJ 07101-1709, USA

10 University of Texas Southwestern Medical School, 5323 Harry Hines Boulevard, Dallas, TX 75390, USA

11 University of Colorado School of Medicine, $13001 \mathrm{E} 17 \mathrm{th} \mathrm{Pl}$, Aurora, CO 80045, USA 


\section{Introduction}

The year 2017, the eighth full year of operation of the Toxicology Investigators Consortium (ToxIC), was marked by a number of achievements and continued robust data collection. A summary of the data collected is provided in the body of this report. Major changes and achievements are described below.

On January 1, 2017, ToxIC moved to the REDCap data collection platform. This provided an even greater level of data security than we had with our previous interface and allowed for the incorporation of two factor authentication of identity before the database was accessed for data entry or searching.

Given the growth of ToxIC and large amount of associated information, policies, and background information, a unique ToxIC website was developed throughout 2016 and went live in 2017 at https://www.toxicregistry.org. The reader of this Annual Report is referred to this website for additional information about ToxIC.

In light of the evolving complexity of ToxIC, it was felt that it was necessary to embark on a long-term strategic plan. That plan was completed in 2017 and its implementation was initiated. Continued implementation is expected throughout 2018. It is hoped that the implementation of the strategic plan will lead to further sophistication of our data collection and analysis, research, post-marketing surveillance, and toxicovigilance activities. It is also hoped that the strategic plan implementation will serve as a direction to ensure the continued long-term sustainability of the ToxIC program.

In 2017, there were 7577 novel cases entered from 40 separate sites comprising 63 separate facilities. The year 2017 saw the closure of three projects, the evolution and growth of existing efforts, and the launch of three of new focused sub-Registries.

A study, in the form of a sub-Registry, on diagnostic modalities in caustic ingestions, which collected data for nearly the entire 8 years of ToxIC's existence, was completed and the sub-Registry was closed by virtue of not being migrated to the REDCap platform. Data analysis collected in this project is now underway.

The focused data collection, also in the form of a subRegistry, on electrocardiographic QRS widening and the antidotal use of sodium bicarbonate was also closed in 2017. The analysis of data from this project is also underway. Hypotheses emerging from this analysis will be potentially tested once this data analysis is completed.

Our data collection of prescription opioid misuse was closed by virtue of it not being migrated to REDCap. With the continued and growing concern about opioid toxicity, it was felt that a new and more robust approach to this critical problem was necessary. The epidemic of opioid abuse and misuse is a major public health concern and new efforts related to this problem by ToxIC are in development. As described below, one such project relating to pediatric opioid exposures has already been instituted.

Our study on the use of lipid resuscitation therapy continues and currently represents the largest known prospective collection of patients treated with this modality. Data analysis for this project is also underway; however, patient accrual is still ongoing. Following our preliminary analysis, the need for objective definable end-points became apparent and were instituted in our mid-2017 database modifications.

The North American Snakebite sub-Registry continues to thrive and currently represents the largest prospective collection of data on these snakebites in existence. The publications and published abstracts from presentations at national professional meetings deriving from this sub-Registry are listed on https://www.toxicregistry.org.

Our extracorporeal substance removal sub-Registry continues to collect unique data on drug clearances by these techniques. Also continuing is the data collection of prognostic factors following drug overdose, now in its fourth year.

Two of our new sub-Registries deal with critical issues related to pediatric exposures: opioids and marijuana. Both of these projects went live with the launch of REDCap on January 1, 2017. Also at that time a sub-Registry on plant and mushroom exposures was initiated. These uncommon but extremely important exposures are very hard to study given the few cases encountered. By aggregating the prospective experience of the network of ToxIC investigators, it is anticipated that a robust series of cases on individual plant and mushroom exposures will be characterized.

There were eight peer-reviewed publications derived from the ToxIC Registry and published by ToxIC investigators in 2017. In addition, there were 17 published abstracts from professional meetings in 2017.

In 2017, ToxIC continued to be supported by a grant on cardiovascular toxicity from the National Institutes of Health, the continuation of our contract with the US Food and Drug Administration, and further unrestricted grant support from BTG International. The latter was used to support the North American Snake Bite Registry.

Ongoing investigator-initiated research projects can be found on the ToxIC website.

This eighth ToxIC Annual Report summarizes the main points of the data collected in our main Registry in 2017. Data from our sub-Registries are published separately.

\section{Methods}

A detailed description of the creation and design of the ToxIC Registry has been previously reported [1]. To be part of the consortium, all medical toxicologists at participating institutions agree to enter data into the ToxIC Registry on all medical 
toxicology consultations performed. Cases are entered on a password-protected encrypted online data collection form. The site uses the REDCap (Research Electronic Data Capture) interface and is hosted by Vanderbilt University. The content of the database is maintained with oversight by the ToxIC Leadership Group. The Registry is compliant with the Health Insurance Portability and Accountability Act and does not collect any protected health information or otherwise identifying fields. Registry participation is pursuant to the participating institutions' Institutional Review Board approval and compliant with their policies and procedures. The Registry has also been independently reviewed by the Western IRB and determined not to meet the threshold of human subject research under federal regulation 45 CFR 46 and associated guidance.

Data collected on each case includes presenting signs and symptoms, clinical course, treatments, limited patient demographics, outcome, laboratory values, and circumstances of, and reasons for, the toxicological exposure. As in prior reports, the term consultation is used in this report to describe any in-person encounter with a medical toxicologist in which a formal evaluation was conducted and placed in the medical record. Such encounters may include admission to a medical toxicology inpatient service, or evaluation by a medical toxicologist as a consulting physician in an emergency department, inpatient unit, or outpatient clinic. The online data collection interface is formatted to ensure data entry remains organized and searchable. Free text entry fields allow providers to provide further detail or supplementary information. As part of the Registry's toxicosurveillance mission, one component of the standard data form is a continuously monitored sentinel detection field that signals novel or unusual cases.

For this report a search of the database was performed to identify cases recorded from January 1, 2017, through December 31, 2017. This descriptive report summarizes case demographics, source and location of consultation, and reasons for encounter and provides case frequencies by individual agent class and treatment provided. We also have included several more focused analyses of particular interest. These dealt with pediatric exposures, marijuana edibles, the use of extracorporeal membrane oxygenation (ECMO), coingestants associated with opioid exposures, and gabapentinoid misuse.

In the tables describing individual agents or agent classes, unless otherwise indicated, cells with fewer than five occurrences were not listed as separate items but are further grouped as "miscellaneous." Percentages noted in tables for individual agents represent their relative proportion within their respective agent class.

For clinical signs or symptoms, the tables provide the percentage of individual signs or symptoms relative to the total number of Registry cases in 2017. Signs and symptoms include the presence or absence of a toxidrome, vital sign abnormalities, and a variety of organ system-based derangements which may arise from a toxic exposure. For each sub- heading in the data collection instrument, investigators are required to either select an abnormality, or "None," to improve the accuracy of data collection and to avoid missing data fields. In the detailed treatment tables, percentages for each treatment modality represent the relative frequency among all treatments rendered.

\section{Results}

In 2017, there were a total of 7577 cases reporting toxicologic exposures to the ToxIC Registry. This is a decrease from the prior 2 years (Fig. 1). Table 1 lists all individual sites that contributed cases in 2017.

\section{Demographics}

Tables 2 and 3 summarize selective demographics including gender, age, race, and ethnicity. In 2017, 50.4\% of cases involved female patients. Sixty patients $(0.8 \%)$ were pregnant (Table 2). New fields were added in 2017 to better represent the population and include transgender patient data. There were 14 male-to-female transgender patients and 22 femaleto-male, making up $0.5 \%$ of patients in the database.

Age distribution in 2017 was similar to past years [2-8]. The majority of patients were adults age 19-65 (63.4\%), followed by adolescents age 13-18 (19.4\%). Children age 12 and under made up $11.8 \%$ of cases.

The most commonly reported race was Caucasian (59.4\%), followed by Black/African (13.2\%) and Asian (4.6\%) (Table 3). Unknown or uncertain race was reported in $19.2 \%$ of cases, similar to 2016 data [8]. Hispanic ethnicity was reported in $11.3 \%$ of cases (Table 3). Nineteen percent of cases reported unknown ethnicity. Race and ethnicity are selfreported by patients, or in cases where a patient is unable to report, it may be reported by the examining medical toxicologist to the best of their ability.

Table 4 summarizes the referral sources of inpatient and outpatient medical toxicology encounters. The majority of inpatient cases $(56.7 \%)$ were generated from the Emergency Department. Only $0.3 \%$ of inpatient encounters were referred from poison centers. Outpatient toxicology evaluations were predominantly referred by primary care or other outpatient physicians $(43.5 \%)$ or were self-referrals $(37.1 \%)$.

Table 5 reports the reasons for medical toxicology encounters. Similar to prior years, intentional pharmaceutical exposures were the most common reason for encounter (54.7\%).

Table 6 presents information on reasons for intentional pharmaceutical exposures. The majority of cases $(67.6 \%)$ were an attempt at self-harm. Of these cases with an attempt at self-harm, $88.2 \%$ represented a suicide attempt. 
Fig. 1 ToxIC Registry total case count by year, $2010-2017$

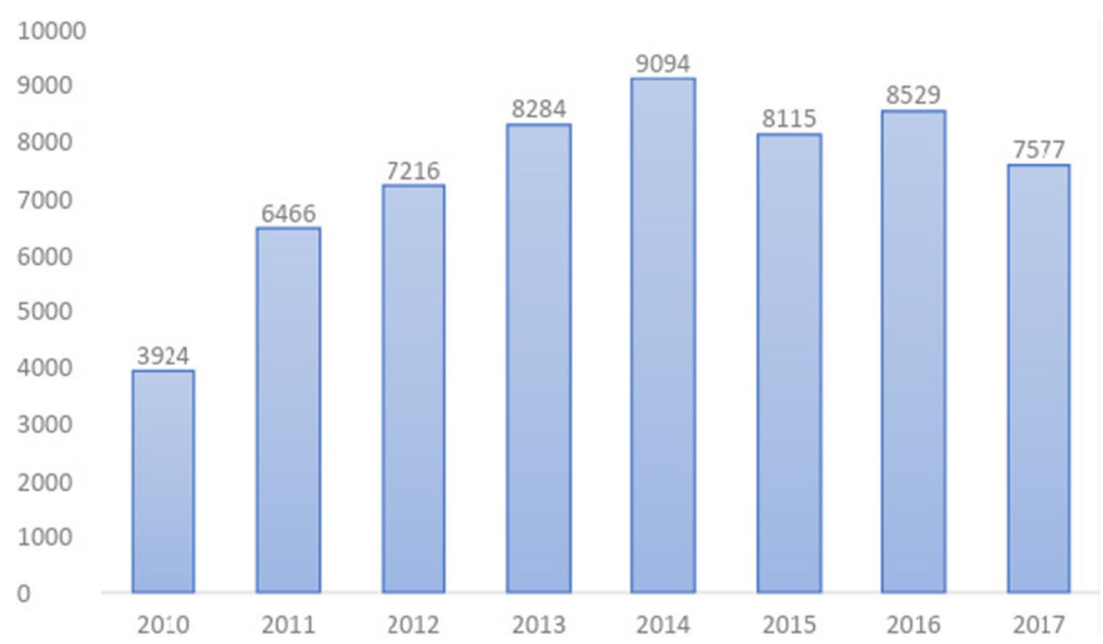

\section{Agent Classes}

Of the 7577 cases entered into the ToxIC Registry in 2017, 2340 cases involved multiple agents for a total of 10,606 individual agent entries. The non-opioid analgesic class was the most common (14.9\%), followed by antidepressants (11.4\%), opioids (10.4\%), and sedative-hypnotic/muscle relaxants (9.2\%). Table 7 presents the totals for each of the 40 agent classes in the Registry.

\section{Pediatric Agent Classes}

Table 8 presents the agent classes reported by age group. There were 3366 individual agent entries in 2017 for 2360 reported pediatric cases with $610(25.8 \%)$ cases involving multiple agents. The top agent classes for all pediatric cases were analgesics (19.5\%), followed by antidepressants (13.0\%) and anticholinergic/antihistamine $(9.2 \%)$. By age category, the most common exposures by agent class were opioids for children $<2$ years old, cardiovascular drugs for ages $2-6$, envenomations for ages 7-12, and analgesics for ages $13-$ 18. Opioids were ranked sixth in agent class frequency for pediatrics with the majority of exposures involving 13-18 year olds, though they were the most common agent class in those under age 2. Envenomations were most commonly reported in the 7-12 year old age category, followed by $13-18$ year olds. Caustic exposures were most often seen in 2-6 year olds.

Table 9 lists the most commonly reported agent classes involved in exposures in children aged 5 or younger. The cardiovascular agent class was the most commonly reported (15.6\%), followed by opioids (8.4\%). Diabetic medications (5.0\%), herbal products/dietary supplements $(3.8 \%)$, and metals (3.8\%) were also reported more frequently in children aged 5 or younger compared to their representation in the Registry as a whole.

\section{Individual Agents by Class}

Tables 10, 11, 12, 13, 14, 15, 16, 17, 18, 19, 20, 21, 23, 24, 25, 26,27 , and 28 present the frequencies of individuals agents, organized by agent class as reported to the Registry in 2017 . The organization follows past years for consistency with three agents - ethanol, lithium, and amphetamine-like hallucinogens - defined as their own agent class, but reported in conjunction with other agent classes (toxic alcohols, anticonvulsants and mood stabilizers, and psychoactives, respectively) for brevity. For agent classes with few overall entries (less than 100), or for which a single agent made up more than $75 \%$ of the cases, or for which the majority of cases were infrequent miscellaneous agents, the results are reported in Tables S1-S16 in the Supplemental Materials.

\section{Analgesics}

Table 10 presents the non-opioid analgesics, the largest class reported in the Registry. Acetaminophen, aspirin, and ibuprofen were the most frequently reported agents in 2017 similar to past years [2-8]. In 2017, gabapentin and pregabalin were moved from the sedative-hypnotics/muscle relaxants class into the analgesics class. In this largest agent class, gabapentin still made up $11.1 \%$ of the category.

\section{Antidepressants}

Table 11 describes the antidepressant agents. The other antidepressant category was again the most frequent, predominantly due to the frequent reporting of bupropion (21.0\%) and trazodone (13.3\%). The selective serotonin reuptake inhibitors (SSRIs), tricyclic antidepressants (TCAs), and serotonin-norepinephrine reuptake inhibitors (SNRIs) were reported with similar agent frequencies to past years $[7,8]$. 
Table 1 Participating institutions providing cases to ToxIC in 2017

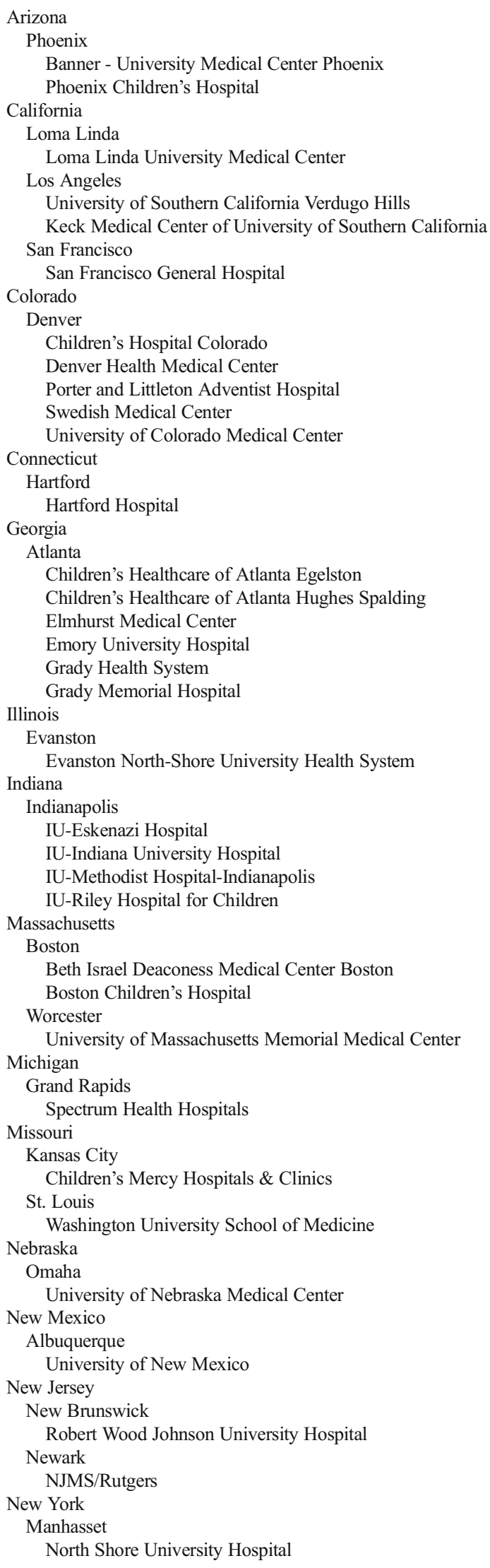

Table 1 (continued)

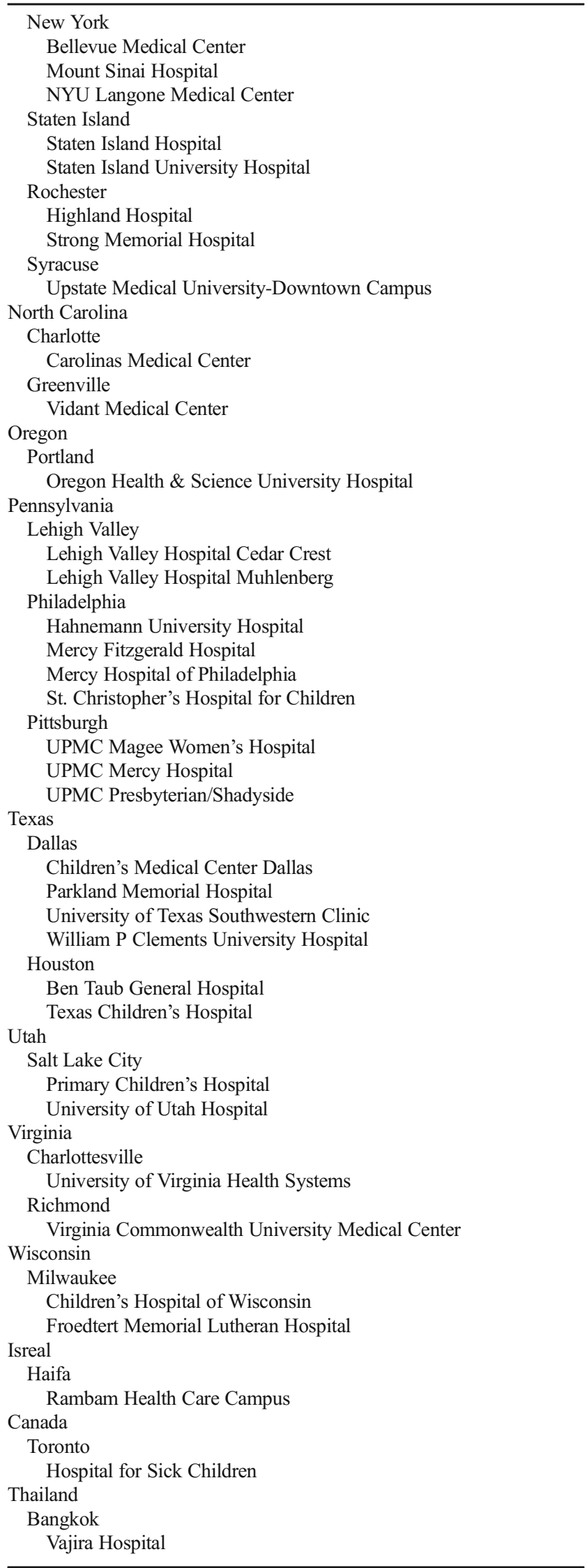


Table 2 ToxIC case demographics - age and gender

\begin{tabular}{ll}
\hline & $N(\%)$ \\
\hline Gender & \\
Male & $3718(49.1)$ \\
Female & $3820(50.4)$ \\
Transgender & \\
$\quad$ Male to female & $14(0.2)$ \\
$\quad$ Female to male & $22(0.3)$ \\
Not recorded & $3(0.0)$ \\
Pregnant & $60(0.8)$ \\
Age (years) & \\
$<2$ & $268(3.5)$ \\
$2-6$ & $371(4.9)$ \\
$7-12$ & $253(3.3)$ \\
$13-18$ & $1468(19.4)$ \\
$19-65$ & $4803(63.4)$ \\
$66-89$ & $376(5.0)$ \\
$>89$ & $13(0.2)$ \\
Unknown & $25(0.3)$ \\
Total & $7577(100)$ \\
\hline
\end{tabular}

\section{Opioids}

Table 12 presents the opioid agent class. As in recent years, heroin was the most commonly reported opioid in the Registry in 2017 (28.9\%). Oxycodone was again the second most frequently reported agent $(14.5 \%)$, though its percent contribution to the class was slightly decreased from last year $(17.8 \%)$

Table 3 ToxIC case demographics — race and Hispanic ethnicity

\begin{tabular}{lc}
\hline & $N(\%)$ \\
\hline Race & \\
Caucasian & $4504(59.4)$ \\
Unknown/uncertain & $1456(19.2)$ \\
Black/African & $999(13.2)$ \\
Asian & $350(4.6)$ \\
Mixed & $136(1.8)$ \\
American Indian/Alaska Native & $111(1.5)$ \\
Other & $16(0.2)$ \\
Native Hawaiian or Pacific Islander & $5(0.1)$ \\
Total & $7577(100)$ \\
Hispanic ethnicity & \\
Hispanic & $854(11.3)$ \\
Non-Hispanic & $5283(69.7)$ \\
Unknown & $1439(19.0)$ \\
Total & $7577(100)$ \\
\hline
\end{tabular}

One case not recorded as Hispanic or non-Hispanic ethnicity

${ }^{\text {a }}$ Hispanic ethnicity as indicated exclusive of race
[8]. Overall, oxycodone has declined in its percent contribution to the opioid agent class each year [2-8]. Tramadol increased slightly from 2016, making up $9.6 \%$ of reported opioids [8]. Reported fentanyl exposures increased by more than $90 \%$ in 2017 making up $7.9 \%$ of the opioid class after being steady at $4.1 \%$ in both 2015 and $2016[7,8]$. In the same years, unspecified opioids had increased from $6.5 \%$ in 2015 to $8.1 \%$ in 2016, but remained fairly stable in 2017 at $7.4 \%$. This increase in fentanyl cases may be a reflection of increased awareness of and testing for fentanyl adulteration of heroin. Additionally, the opioid unspecified class which has had a trending increase since the Registry began in 2010, may be capturing additional cases of adulterated heroin when testing is not available [2-8]. In 2017, the miscellaneous opioids included more specific designer opioids not previously reported to the Registry including butanoyl-4-fluorofentanyl, fluorofentanyl, fluoroisobutyryl fentanyl (4- or para-), methyl norfentanyl, $\mathrm{N}$-allyl norfentanyl, butyrylfentanyl (butyr-), and carfentanil. The designer opioid U47700 and methylfentanyl were also reported after being reported to the Registry for the first time in 2016.

\section{Opioid Coingestants}

In 2017, there were 988 total opioid agents reported in 718 unique case entries. The frequency of coingestion was high with $403(56.1 \%)$ out of 718 unique cases having more than one primary agent reported. Coingestion rates varied widely; however, based on specific opioid ingested, ranging from a high of $84.4 \%$ of cases for which hydrocodone was reported to a low of $21 \%$ when buprenorphine was reported. There were 270 cases $(37.6 \%)$ with more than one opioid reported as a primary agent. Heroin was the most common opioid reported in 2017 with 318 case entries. The most common coingestions included stimulants occurring in $72(22.6 \%)$ of heroin cases. Cocaine was the most commonly reported stimulant reported with heroin occurring in 49 (15.4\%) followed by methamphetamine in $23(7.2 \%)$. Alprazolam was the most commonly reported benzodiazepine reported with heroin occurring in 14 $(4.4 \%)$. Other opioids were reported in $26(8.2 \%)$ of cases involving heroin. Tramadol was the second most commonly reported opioid in 2017 occurring in 104 case entries. Coingestion was reported in $34(32.7 \%)$ of tramadol cases, and while there were some drugs reported more than once, coingestion involved a variety of other agents. There were 18 unique agents reported along with tramadol; THC, kratom, gabapentin, cyclobenzaprine, and alcohol were all reported more than twice. Oxycodone was reported in 100 case entries with coingestion occurring in 57 (57.0\%). Acetaminophen was the most commonly reported drug ingested with oxycodone occurring in 13 cases (13.0\%). Benzodiazepines were reported as coingestants in 22 
(22.0\%) of oxycodone cases and alprazolam was the most commonly reported benzodiazepine in 11 (11.0\%) of cases. Fentanyl was reported in 87 case entries with 53 cases $(60.9 \%)$ involving coingestion and cocaine was the single most common drug, occurring in 13 cases (14.9\%). Opioids were the most frequent class of drug reported as coingestion for fentanyl reported for nearly a third of all fentanyl cases with co ingestion. Methadone was reported in 79 case entries with coingestion in 30 (37.9\%) and other opioids reported most commonly occurring in 19 cases $(24.1 \%)$ with heroin being the most common opioid reported with methadone. Other drugs reported with methadone included cocaine in $8(10.1 \%)$ and alprazolam was the most commonly reported benzodiazepine reported in 5 cases $(6.3 \%)$. Hydrocodone was reported in 77 cases with $39(50.6 \%)$ involving coingestion with acetaminophen as ingestion of the coformulation products for hydrocodone and acetaminophen were common. Sedative ingestion was common with hydrocodone though a variety of sedatives were reported including gabapentin, clonazepam, lorazepam, baclofen, and alcohol and although alprazolam was

Table 4 ToxIC registry case referral sources by inpatient/outpatient status

\begin{tabular}{ll}
\hline & $N(\%)$ \\
\hline Emergency Department (ED) or inpatient (IP) $^{\mathrm{a}}$ & \\
ED & $4066(56.7)$ \\
Admitting service & $1726(24.1)$ \\
Outside hospital transfer & $932(13.0)$ \\
Request from another hospital service (not ED) & $239(3.3)$ \\
Self-referral & $180(2.5)$ \\
Poison Center & $24(0.3)$ \\
Primary care provider or other outpatient treating & $4(0.1)$ \\
physician & $2(0.0)$ \\
Employer/Independent medical evaluation & $7173(100)$ \\
ED/IP total & \\
Outpatient (OP)/clinic/office consultation & \\
Primary care provider or other OP physician & $175(43.5)$ \\
Self-referral & $149(37.1)$ \\
Employer/Independent medical evaluation & $35(8.7)$ \\
Poison Center & $24(6.0)$ \\
ED & $10(2.5)$ \\
Admitting service & $3(0.7)$ \\
Outside hospital transfer & $3(0.7)$ \\
Request from another hospital service (not ED) & $3(0.7)$ \\
OP total & $402(100)$ \\
\hline
\end{tabular}

Two cases not recorded inpatient or outpatient location

${ }^{\text {a }}$ Percentage based on the total number of cases $(N=7173)$ seen by a medical toxicologist as consultant (ED or IP) or as attending (IP)

${ }^{\mathrm{b}}$ Percentage based on the total number of cases $(N=402)$ seen by a medical toxicologist as outpatient, clinic visit, or office consultation
Table 5 Reason for medical toxicology encounter

\begin{tabular}{ll}
\hline & $N(\%)$ \\
\hline Intentional exposure — pharmaceutical & $4144(54.7)$ \\
Intentional exposure — non-pharmaceutical & $921(12.2)$ \\
Unintentional exposure — pharmaceutical & $610(8.1)$ \\
Unintentional exposure — non-pharmaceutical & $322(4.2)$ \\
Envenomation —-snake & $297(3.9)$ \\
Organ system dysfunction & $256(3.4)$ \\
Withdrawal —opioid & $217(2.9)$ \\
Withdrawal—ethanol & $149(2.0)$ \\
Ethanol abuse & $132(1.7)$ \\
Interpretation of toxicology data & $123(1.6)$ \\
Environmental evaluation & $122(1.6)$ \\
Occupational evaluation & $80(1.1)$ \\
Envenomation—spider & $55(0.7)$ \\
Malicious/criminal & $41(0.5)$ \\
Envenomation—other & $38(0.5)$ \\
Withdrawal—sedative-hypnotic & $35(0.5)$ \\
Withdrawal—other & $16(0.2)$ \\
Withdrawal—cocaine/amphetamine & $6(0.1)$ \\
Envenomation—scorpion & $5(0.1)$ \\
Marine/fish poisoning & $5(0.1)$ \\
Not recorded & $3(0.0)$ \\
Total & $7577(100)$ \\
\hline
\end{tabular}

the most common. Buprenorphine was reported in 66 cases with $16(24.2 \%)$ having coingestion reported. Other opiates, sedatives, cocaine, and benzodiazepines were the most commonly reported substances with buprenorphine. Morphine was reported in 37 cases and 26 (70.3\%) had coingestion with opioid coingestion occurring in 10 and oxycodone being the most commonly reported other opioid along with morphine. Non-specific opioids (opioids NOS) were reported in 82 unique entries and 13 cases included co ingestion with sedatives being reported in almost half of these cases. Several different benzodiazepines, alcohol, and gabapentin were reported when non-specified opioid ingestion occurred in case entries.

Overall, alprazolam, followed by clonazepam, were the most common benzodiazepines reported with an opioid reported as primary agent. Illicit opioids had other illicit drugs including cocaine, methamphetamine, fentanyl, or heroin reported as coingestion though commonly abused pharmaceuticals were also not uncommon for illicit opioid cases (e.g., sedatives including alprazolam, gabapentin, and baclofen). Coingestion with other opioids and sedatives was also commonly reported for the pharmaceutical opioids as were illicit drugs, though this was at lower rates than their illicit opioid counterparts. 


\section{Sedative-Hypnotics/Muscle Relaxants}

Table 13 presents the sedative-hypnotics/muscle relaxants class. Benzodiazepines remained the most commonly reported subclass of sedative-hypnotics/muscle relaxants in 2017. Alprazolam (22.8\%) and clonazepam (18.6\%) were again the most frequent agents both for the benzodiazepine subclass, as well as for the agent class as a whole. Of note, in 2016, gabapentin was reclassified from the sedative-hypnotics/muscle relaxants class to the non-opioid analgesic class, so is summarized in Table 10. The number of gabapentin cases was approximately stable from 2016. The muscle relaxants baclofen and cyclobenzaprine were reported with equal frequency $(10.0 \%)$ in 2017 . Barbiturates were again infrequently reported with butalbital making up the majority of these $(1.8 \%)$.

\section{Toxic Alcohols and Ethanol}

Table 14 presents data on ethanol and toxic alcohols. As in prior years, ethanol is considered its own agent class. There were 723 ethanol exposures in 2017. Among the non-ethanol alcohols and glycols, isopropanol (37.3\%) and ethylene glycol $(34.7 \%)$ were similarly reported.

\section{Anticholinergics}

Table 15 shows the anticholinergics and antihistamines agent class. As in past years, diphenhydramine $(59.1 \%$ ) made up the majority of the agent class, with hydroxyzine $(14.9 \%)$ following.

Table 6 Detailed reason for encounter-intentional pharmaceutical exposure

\begin{tabular}{ll}
\hline & $N(\%)$ \\
\hline Reason for intentional pharmaceutical exposure subgroup ${ }^{\mathrm{a}}$ \\
Attempt at self-harm & $2803(67.6)$ \\
Misuse/abuse & $718(17.3)$ \\
Therapeutic use & $328(7.9)$ \\
Unknown & $294(7.1)$ \\
Not recorded & $1(0.0)$ \\
Total & $4144(100)$ \\
Attempt at self-harm- suicidal intent subclassification & \\
Suicidal intent & $2473(88.2)$ \\
Suicidal intent unknown & $248(8.8)$ \\
No suicidal intent & $79(2.8)$ \\
Not recorded & $3(0.1)$ \\
Total & $2803(100)$ \\
\hline
\end{tabular}

${ }^{a}$ Percentage of total number of cases $(N=4144)$ indicating primary reason for encounter due to intentional pharmaceutical exposure

${ }^{\mathrm{b}}$ Percentage of number of cases indicating attempt at self-harm $(N=$ 2803)
Table 7 Agent classes involved in medical toxicology consultation

\begin{tabular}{|c|c|}
\hline & $N(\%)^{\mathrm{a}}$ \\
\hline Analgesic & $1582(14.9)$ \\
\hline Antidepressant & $1207(11.4)$ \\
\hline Opioid & $1101(10.4)$ \\
\hline Sedative-hypnotic/muscle relaxant & $972(9.2)$ \\
\hline Ethanol & $723(6.8)$ \\
\hline Anticholinergic/antihistamine & $669(6.3)$ \\
\hline Sympathomimetic & $658(6.2)$ \\
\hline Cardiovascular & $597(5.6)$ \\
\hline Antipsychotic & $556(5.2)$ \\
\hline Anticonvulsant & $399(3.8)$ \\
\hline Envenomation and marine & $375(3.5)$ \\
\hline Psychoactive & $223(2.1)$ \\
\hline Diabetic medication & $172(1.6)$ \\
\hline Lithium & $121(1.1)$ \\
\hline Toxic alcohol & $118(1.1)$ \\
\hline Metals & $115(1.1)$ \\
\hline Cough and cold products & $112(1.1)$ \\
\hline Herbal products/dietary supplements & $111(1.0)$ \\
\hline Gases/irritants/vapors/dusts & $94(0.9)$ \\
\hline Caustic & $89(0.8)$ \\
\hline Hydrocarbon & $75(0.7)$ \\
\hline Household products & $68(0.6)$ \\
\hline Plants and fungi & $65(0.6)$ \\
\hline Antimicrobial & $60(0.6)$ \\
\hline Endocrine & $38(0.4)$ \\
\hline Chemotherapeutic/immunological & $35(0.3)$ \\
\hline Other non-pharmaceutical product & $33(0.3)$ \\
\hline Gastrointestinal agents & $30(0.3)$ \\
\hline Anesthetic & $27(0.3)$ \\
\hline Insecticide & $27(0.3)$ \\
\hline Anticoagulant & $24(0.2)$ \\
\hline Other pharmaceutical product & $18(0.2)$ \\
\hline Amphetamine-like hallucinogen & $12(0.1)$ \\
\hline Herbicide & $10(0.1)$ \\
\hline WMD/riot agent/radiological & $7(0.1)$ \\
\hline Anti-parkinsonism drugs & $6(0.1)$ \\
\hline Rodenticide & $6(0.1)$ \\
\hline Ingested foreign body & $5(0.0)$ \\
\hline Pulmonary & $4(0.0)$ \\
\hline Fungicide & NR $(0.0)$ \\
\hline Unknown agent & $62(0.6)$ \\
\hline Total & $10,606(100)$ \\
\hline
\end{tabular}


Table 8 ToxIC 2017—agent classes for pediatric cases by age group

\begin{tabular}{|c|c|c|c|c|c|c|c|}
\hline & Exposure rank & Totals & $\%^{\mathrm{a}}$ & Age $<2$ & Age $2-6$ & $\begin{array}{l}\text { Age 7- } \\
12\end{array}$ & $\begin{array}{l}\text { Age 13- } \\
18\end{array}$ \\
\hline Analgesic & 1 & 658 & $19.5 \%$ & 23 & 20 & 30 & 585 \\
\hline Antidepressant & 2 & 436 & $13.0 \%$ & 13 & 25 & 20 & 378 \\
\hline Anticholinergic/antihistamine & 3 & 309 & $9.2 \%$ & 10 & 24 & 30 & 245 \\
\hline Unknown/blank & 4 & 234 & $7.0 \%$ & 36 & 62 & 36 & 100 \\
\hline Cardiovascular & 5 & 224 & $6.7 \%$ & 36 & 82 & 25 & 81 \\
\hline Opioid & 6 & 172 & $5.1 \%$ & 37 & 20 & 5 & 110 \\
\hline Envenomation & 7 & 171 & $5.1 \%$ & 6 & 37 & 71 & 57 \\
\hline Antipsychotic & 8 & 158 & $4.7 \%$ & 5 & 10 & 8 & 135 \\
\hline Sympathomimetic & 9 & 155 & $4.6 \%$ & 31 & 24 & 12 & 88 \\
\hline Sedative-hypnotic/muscle relaxant & 10 & 148 & $4.4 \%$ & 9 & 16 & 14 & 109 \\
\hline Anticonvulsant & 11 & 110 & $3.3 \%$ & 8 & 16 & 14 & 72 \\
\hline Psychoactive & 12 & 67 & $2.0 \%$ & 8 & 14 & 3 & 42 \\
\hline Herbal/dietary supp/vitamins & 13 & 61 & $1.8 \%$ & 9 & 17 & 4 & 31 \\
\hline Diabetic med & 14 & 59 & $1.8 \%$ & 14 & 23 & 1 & 21 \\
\hline Ethanol & 14 & 59 & $1.8 \%$ & 5 & 2 & 2 & 50 \\
\hline Cough/cold & 15 & 50 & $1.5 \%$ & 1 & 7 & 2 & 40 \\
\hline Metals & 16 & 48 & $1.4 \%$ & 8 & 19 & 5 & 16 \\
\hline Household product & 17 & 26 & $0.8 \%$ & 11 & 6 & 1 & 8 \\
\hline Hydrocarbons & 18 & 24 & $0.7 \%$ & 13 & 9 & 0 & 2 \\
\hline Lithium & 19 & 23 & $0.7 \%$ & 1 & 1 & 2 & 19 \\
\hline Antimicrobial & 20 & 22 & $0.7 \%$ & 4 & 3 & 2 & 13 \\
\hline Caustic & 21 & 19 & $0.6 \%$ & 5 & 10 & 1 & 3 \\
\hline Toxic alcohols & 22 & 18 & $0.5 \%$ & 5 & 3 & 2 & 8 \\
\hline Endocrine & 23 & 16 & $0.5 \%$ & 1 & 7 & 1 & 7 \\
\hline GI Agent & 24 & 15 & $0.4 \%$ & 3 & 2 & 1 & 9 \\
\hline Chemotherapeutic/immune & 25 & 14 & $0.4 \%$ & 3 & 2 & 3 & 6 \\
\hline Gases/vapors/irritants/dusts & 25 & 14 & $0.4 \%$ & 5 & 2 & 3 & 4 \\
\hline Plants/fungi & 26 & 12 & $0.4 \%$ & 3 & 4 & 3 & 2 \\
\hline Anesthetic & 27 & 10 & $0.3 \%$ & 3 & 1 & 2 & 4 \\
\hline Other non-pharmaceutical & 28 & 9 & $0.3 \%$ & 2 & 2 & 1 & 4 \\
\hline Other pharmaceutical & 29 & 7 & $0.2 \%$ & 1 & 1 & 0 & 5 \\
\hline Amphetamine-like hallucinogen & 30 & 4 & $0.1 \%$ & 0 & 1 & 1 & 2 \\
\hline Anticoagulant & 31 & 3 & $0.1 \%$ & 2 & 1 & 0 & 0 \\
\hline Ingested foreign body & 31 & 3 & $0.1 \%$ & 1 & 1 & 0 & 1 \\
\hline Insecticide & 32 & 2 & $0.1 \%$ & 2 & 0 & 0 & 0 \\
\hline Pulmonary & 32 & 2 & $0.1 \%$ & 0 & 1 & 0 & 1 \\
\hline Rodenticide & 32 & 2 & $0.1 \%$ & 1 & 1 & 0 & 0 \\
\hline Parkinson's med & 33 & 1 & $0.0 \%$ & 0 & 1 & 0 & 0 \\
\hline WMD & 33 & 1 & $0.0 \%$ & 1 & 0 & 0 & 0 \\
\hline Totals & & 3366 & $100.0 \%$ & & & & \\
\hline
\end{tabular}

${ }^{a}$ Percentages are out of total number of reported agent entries per year; 610 cases $(25.8 \%)$ reported multiple agents

\section{Sympathomimetics}

Table 16 summarizes the sympathomimetics agent class. Cocaine $(38.8 \%)$ was the leading agent reported. Methamphetamine (27.4\%) and amphetamine $(9.6 \%)$ were the next most commonly reported, consistent with prior years.

\section{Cardiovascular Agents}

Table 17 presents the cardiovascular agent class. In 2017, the sympatholytic subclass was the most commonly reported (25.1\%), outnumbering beta blockers $(23.3 \%)$ for the first time in the Registry. Among all of the cardiovascular agents, 
Table 9 Most frequent exposures by agent class- age $\leq 5$ years

\begin{tabular}{ll}
\hline & $N(\%)^{\mathrm{a}}$ \\
\hline Cardiovascular & $106(15.6)$ \\
Opioid & $57(8.4)$ \\
Sympathomimetic & $52(7.7)$ \\
Analgesic & $43(6.3)$ \\
Envenomation & $38(5.6)$ \\
Antidepressant & $34(5.0)$ \\
Diabetic medication & $34(5.0)$ \\
Anticholinergic/antihistamine & $33(4.9)$ \\
Herbal products/dietary supplements & $26(3.8)$ \\
Metals & $26(3.8)$ \\
Sedative-hypnotics/muscle relaxant & $24(3.5)$ \\
Anticonvulsant & $24(3.5)$ \\
Psychoactive & $22(3.2)$ \\
Hydrocarbon & $22(3.2)$ \\
Household & $17(2.5)$ \\
Antipsychotic & $15(2.2)$ \\
Caustic & $15(2.2)$ \\
Class total & $678(100)$ \\
\hline
\end{tabular}

${ }^{\text {a }}$ Percentages are out of total number of agent exposures reported in children aged 5 or younger in $2017(N=678)$

clonidine was the most commonly reported agent (18.9\%), followed by amlodipine (9.9\%), lisinopril (8.4\%), and metoprolol $(7.7 \%)$. Other cardiovascular agent subclasses - other antihypertensives and vasodilators, antidysrhythmics and other cardiovascular agents, cardiac glycosides, diuretics, and angiotensin receptor blockers - each made a smaller contribution to the class, altogether accounting for about one-quarter of the agents.

Table 10 Analgesics

\begin{tabular}{lc}
\hline & $N(\%)$ \\
\hline Acetaminophen & $901(57.0)$ \\
Aspirin & $202(12.8)$ \\
Ibuprofen & $183(11.6)$ \\
Gabapentin & $176(11.1)$ \\
Naproxen & $57(3.6)$ \\
Pregabalin & $25(1.6)$ \\
Salicylic acid & $10(0.6)$ \\
Analgesic unspecified & $6(0.4)$ \\
Meloxicam & $5(0.3)$ \\
Miscellaneous & a \\
Class total & $17(1.1)$ \\
\hline
\end{tabular}

${ }^{\text {a }}$ Includes aminophenazone, diclofenac, etoricoxib, ketorolac, mefenamic acid, unspecified NSAID, other analgesic, phenylbutazone, salicylamide, and salsalate
Table 11 Antidepressants

\begin{tabular}{|c|c|}
\hline & $N(\%)$ \\
\hline Other antidepressants & $476(39.4)$ \\
\hline Bupropion & $252(21.0)$ \\
\hline Trazodone & $161(13.3)$ \\
\hline Mirtazapine & $43(3.6)$ \\
\hline Vilazodone & $9(0.7)$ \\
\hline Antidepressant unspecified & $8(0.7)$ \\
\hline Miscellaneous $^{\mathrm{a}}$ & $<5(<0.4)$ \\
\hline Selective serotonin reuptake inhibitors (SSRIs) & $445(36.9)$ \\
\hline Sertraline & $128(10.6)$ \\
\hline Fluoxetine & $116(9.6)$ \\
\hline Citalopram & $96(8.0)$ \\
\hline Escitalopram & $80(6.6)$ \\
\hline Paroxetine & $25(2.1)$ \\
\hline Tricyclic antidepressants (TCAs) & $166(13.8)$ \\
\hline Amitriptyline & $105(8.7)$ \\
\hline Nortriptyline & $28(2.3)$ \\
\hline Doxepin & $25(2.1)$ \\
\hline Miscellaneous $^{\mathrm{b}}$ & $8(0.7)$ \\
\hline Serotonin-norepinephrine reuptake inhibitors (SNRIs) & $120(9.9)$ \\
\hline Venlafaxine & $80(6.6)$ \\
\hline Duloxetine & $30(2.5)$ \\
\hline Desvenlafaxine & $6(0.5)$ \\
\hline Miscellaneous $^{\mathrm{c}}$ & $<5(<0.4)$ \\
\hline Class Total & $1207(100)$ \\
\hline
\end{tabular}

\footnotetext{
${ }^{\mathrm{a}}$ Includes phenelzine and vortioxetine

${ }^{\mathrm{b}}$ Includes imipramine, clomipramine, amoxapine, and dosulepin

${ }^{\mathrm{c}}$ Includes fluvoxamine
}

\section{Antipsychotics}

Table 18 shows the antipsychotic agent class. Distribution of agents was similar to prior years. Quetiapine made up early half of the agent class $(47.5 \%)$. Olanzapine was the next most common $(14.6 \%)$, followed by risperidone $(7.2 \%)$ and aripiprazole $(7.0 \%)$.

\section{Anticonvulsants, Mood Stabilizers, and Lithium}

Table 19 presents the anticonvulsants and mood stabilizers, along with lithium. As in past years, lithium is considered its own agent class, but for brevity, is presented along with the anticonvulsants and mood stabilizers. There were 121 cases reporting lithium exposure in 2017. The distribution of anticonvulsants and mood stabilizers followed a similar trend to past years. Lamotrigine was the most common agent in the class $(27.1 \%)$, followed by valproic acid (22.8\%). Carbamazepine and topiramate were equally reported $(9.5 \%)$. 
Table 12 Opioids

\begin{tabular}{ll}
\hline & $N(\%)$ \\
\hline Heroin & $318(28.9)$ \\
Oxycodone & $160(14.5)$ \\
Tramadol & $106(9.6)$ \\
Fentanyl & $87(7.9)$ \\
Opioid unspecified & $82(7.4)$ \\
Methadone & $79(7.2)$ \\
Hydrocodone & $78(7.1)$ \\
Buprenorphine & $67(6.1)$ \\
Morphine & $38(3.5)$ \\
Hydromorphone & $17(1.5)$ \\
Codeine & $15(1.4)$ \\
Loperamide & $12(1.1)$ \\
Naltrexone & $9(0.8)$ \\
Oxymorphone & $6(0.5)$ \\
Miscellaneous & a \\
Class total & $27(2.5)$ \\
\hline
\end{tabular}

${ }^{a}$ Includes U47700 (designer opioid), butanoyl-4-fluorofentanyl, methylfentanyl (3- or alpha), diphenoxylate, fluorofentanyl, fluoroisobutyryl fentanyl (4- or para-), methyl norfentanyl, N-allyl norfentanyl, butyrylfentanyl (butyr-), carfentanil, naloxone, normethadone, opium (raw, latex), Papaver somniferum (plant parts), and tapentadol

\section{Envenomations and Marine Poisonings}

Table 20 summarizes the envenomations and marine poisonings. Agkistrodon species exposures slightly outnumbered Crotalus species exposures in 2017 (28.8\% vs 25.3\%). Unspecified snake exposures were the next most common $(10.1 \%)$. Loxosceles exposures increased from 2016 when they made up $4.2 \%$ of the agent class, making up $8.5 \%$ of the class in 2017 [8]. Chilopoda species exposures also were reported more frequently than in past years with 15 cases, making up $4 \%$ of the agent class.

\section{Psychoactives}

Table 21 presents the psychoactive agents and the amphetaminelike hallucinogen methylenedioxymethamphetamine (molly). Molly was reported in 12 cases in 2017, an increase from 2016 when 6 cases were reported [7]. In 2017, the number of marijuana cases surpassed the number of synthetic cannabinoids after a 2-year trend of synthetic cannabinoids being reported more frequently $[7,8]$.

\section{Marijuana Edibles}

Figure 2 presents annual data on exposures to marijuana edible agents from 2012 through 2017, reported by age range. In
Table 13 Sedative-hypnotics/muscle relaxants by sub-type

\begin{tabular}{|c|c|}
\hline & $N(\%)$ \\
\hline Benzodiazepines & $571(58.8)$ \\
\hline Alprazolam & $220(22.8)$ \\
\hline Clonazepam & $181(18.6)$ \\
\hline Lorazepam & $80(8.2)$ \\
\hline Diazepam & $42(4.3)$ \\
\hline Benzodiazepine unspecified & $23(2.4)$ \\
\hline Temazepam & $11(1.1)$ \\
\hline Chlordiazepoxide & $6(0.6)$ \\
\hline Miscellaneous $^{\mathrm{a}}$ & $8(0.8)$ \\
\hline Muscle relaxants & $259(26.7)$ \\
\hline Baclofen & $97(10.0)$ \\
\hline Cyclobenzaprine & $97(10.0)$ \\
\hline Carisoprodol & $22(2.3)$ \\
\hline Tizanidine & $22(2.3)$ \\
\hline Methocarbamol & $15(1.5)$ \\
\hline Metaxalone & $5(0.5)$ \\
\hline Miscellaneous ${ }^{\mathrm{b}}$ & $<5(<0.5)$ \\
\hline Non-benzodiazepine agonists (“Z” drugs) & $63(6.5)$ \\
\hline Zolpidem & $58(6.0)$ \\
\hline Miscellaneous $^{\mathrm{c}}$ & $5(0.5)$ \\
\hline Other Sedatives & $52(5.3)$ \\
\hline Sedative-hypnotic unspecified & $19(1.9)$ \\
\hline Buspirone & $17(1.8)$ \\
\hline Phenibut & $5(0.5)$ \\
\hline Miscellaneous $^{\mathrm{d}}$ & $11(1.1)$ \\
\hline Barbiturates & $27(2.8)$ \\
\hline Butalbital & $17(1.8)$ \\
\hline Phenobarbital & $7(0.7)$ \\
\hline Miscellaneous $^{\mathrm{e}}$ & $3(0.3)$ \\
\hline Class total & $972(100)$ \\
\hline
\end{tabular}

a Includes clorazepate, midazolam, flunitrazepam, and oxazepam

${ }^{\mathrm{b}}$ Includes orphenadrine

${ }^{\mathrm{c}}$ Includes eszopiclone and zopiclone

${ }^{\mathrm{d}}$ Includes propofol, chlorzoxazone, etizolam, and clomethiazole

${ }^{\mathrm{e}}$ Includes butabarbital and barbiturate unspecified

2012, there were no cases of oral marijuana ingestion in children aged $0-6$. There were 10 cases reported in this age group in 2017 and 8 in 2017. Use among those aged 66-89 was low with only 1 case reported over the timeframe.

\section{Pediatric Exposures to Drugs of Abuse}

Tables $22 \mathrm{~A}-\mathrm{J}$ present more detailed information about cases involving pediatric exposures to agents that are commonly used as drugs of abuse. If a case was identified reporting a drug of abuse, then additional agents in a polypharmacy exposure were also included in the analysis. Overall, 123 cases 
Table 14 Ethanol and toxic alcohols

\begin{tabular}{ll}
\hline & $N(\%)$ \\
\hline Ethanol $^{\mathrm{a}}$ & $723(100)$ \\
Non-ethanol alcohols and glycols & \\
Isopropanol & $44(37.3)$ \\
Ethylene glycol & $41(34.7)$ \\
Methanol & $18(15.3)$ \\
Acetone & $5(4.2)$ \\
Miscellaneous & $\mathrm{b}$ \\
Class total & $10(8.5)$ \\
\hline
\end{tabular}

${ }^{a}$ Ethanol is considered a separate agent class

${ }^{\mathrm{b}}$ Includes benzyl alcohol, butyl ethylene glycol, diethyl ether, diethylene glycol, dipropylene glycol, glycol ethers, propylene glycol, and toxic alcohol unspecified

involving pediatrics exposed to drugs of abuse were identified. Tables $22 \mathrm{~A}, \mathrm{~B}, \mathrm{C}$, and D present the distribution of these patients by overall race and ethnicity, as well as race and ethnicity broken down by gender. Overall, $59.3 \%$ of patients were Caucasian and $21.1 \%$ were African American. Tables 22 $\mathrm{E}$ and $\mathrm{F}$ compare the numbers of single agent exposures and multiple agent exposures overall and by gender. Single agent exposures were more common (61.8\%). Table $22 \mathrm{G}$ and $\mathrm{H}$ show the most common agent classes involved in these exposures overall and by gender. The top agent classes were psychoactives, sympathomimetics, opioids, and antidepressants. These remained similarly reported among both male and female genders. Table $22 \mathrm{I}$ and $\mathrm{J}$ show the reasons for medical

Table 15 Anticholinergics and antihistamines

\begin{tabular}{ll}
\hline & $N(\%)$ \\
\hline Diphenhydramine & $413(61.7)$ \\
Hydroxyzine & $85(12.7)$ \\
Doxylamine & $32(4.8)$ \\
Chlorpheniramine & $22(3.3)$ \\
Benztropine & $21(3.1)$ \\
Cetirizine & $18(2.7)$ \\
Promethazine & $14(2.1)$ \\
Loratadine & $9(1.3)$ \\
Trihexyphenidyl & $8(1.2)$ \\
Dicyclomine & $7(1.0)$ \\
Dimenhydrinate & $7(1.0)$ \\
Meclizine & $5(0.7)$ \\
Miscellaneous & a \\
Class total & $28(4.2)$ \\
\hline
\end{tabular}

${ }^{a}$ Includes anticholinergic unspecified, antihistamine unspecified, atropine, brompheniramine, buclizine, cyproheptadine, fexofenadine, homatropine, hyoscyamine, mirabegron, oxybutynin, pheniramine, pyrilamine, scopolamine, and triprolidine
Table 16 Sympathomimetics

\begin{tabular}{ll}
\hline & $N(\%)$ \\
\hline Cocaine & $255(38.8)$ \\
Methamphetamine & $180(27.4)$ \\
Amphetamine & $63(9.6)$ \\
Methylphenidate & $36(5.5)$ \\
Dextroamphetamine & $30(4.6)$ \\
Lisdexamfetamine & $16(2.4)$ \\
Sympathomimetic unspecified & $15(2.3)$ \\
Methylenedioxy- $N$-methamphetamine & $10(1.5)$ \\
Pseudoephedrine & $9(1.4)$ \\
Phentermine & $8(1.2)$ \\
Atomoxetine & $7(1.1)$ \\
Dexmethylphenidate & $5(0.8)$ \\
Phenylephrine & $5(0.8)$ \\
Miscellaneous &
\end{tabular}

${ }^{\mathrm{a}}$ Includes clenbuterol, epinephrine, 2C series drugs, 25I-NBOMe, 3fluoroethamphetamine, 4-fluoroamphetamine, butylone, cathinone, ephedrine, ethylphenidate, isometheptine, MDPV, norpseudoephedrine, phenylpropanolamine, and tetrahydrozoline

toxicology encounters overall and broken down by gender. Intentional non-pharmaceutical exposures were the most commonly reported (38.2\%), followed by intentional pharmaceutical exposures $(27.6 \%)$.

\section{Diabetic Agents}

Table 23 shows diabetic medications. There were 172 diabetic medications reported to the Registry. Metformin was the most commonly reported agent (33.1\%). In 2017, glipizide (23.3\%) was reported more frequently than insulin $(22.1 \%)$, a change from prior years.

\section{Metals}

Table 24 presents the metal agent class. There were 115 agents reported in 2017, with the top agents and frequencies similar to past years. Lead was the most commonly reported $(27.8 \%)$, followed by iron $(22.6 \%)$. Cobalt $(8.7 \%)$ and chromium $(5.2 \%)$ entries have continued to decrease since they peaked in 2011, coincident with the discontinued use of certain metal-on-metal hip joint prostheses [2-8].

\section{Herbal Products and Dietary Supplements}

Table 25 shows the herbal products and dietary supplements agent class. Similar to past years, this category included a diverse group of products, many of which were infrequently 
Table 17 Cardiovascular agents by sub-type

\begin{tabular}{|c|c|}
\hline & $N(\%)$ \\
\hline Sympatholytics & $150(25.1)$ \\
\hline Clonidine & $113(18.9)$ \\
\hline Guanfacine & $34(5.7)$ \\
\hline Miscellaneous $^{\mathrm{a}}$ & $<5(<0.8)$ \\
\hline Beta blockers & $139(23.3)$ \\
\hline Metoprolol & $46(7.7)$ \\
\hline Propranolol & $39(6.5)$ \\
\hline Carvedilol & $24(4.0)$ \\
\hline Atenolol & $19(3.2)$ \\
\hline Labetalol & $9(1.5)$ \\
\hline Miscellaneous $^{\mathrm{b}}$ & $<5(<0.8)$ \\
\hline Calcium channel antagonists & $104(17.4)$ \\
\hline Amlodipine & $59(9.9)$ \\
\hline Diltiazem & $24(4.0)$ \\
\hline Verapamil & $10(1.7)$ \\
\hline Nifedipine & $9(1.5)$ \\
\hline Miscellaneous ${ }^{\mathrm{c}}$ & $<5(<0.8)$ \\
\hline ACE inhibitors & $58(9.7)$ \\
\hline Lisinopril & $50(8.4)$ \\
\hline Enalapril & $5(0.8)$ \\
\hline Miscellaneous $^{\mathrm{d}}$ & $<5(<0.8)$ \\
\hline Other antihypertensives and vasodilators & $40(6.7)$ \\
\hline Prazosin & $26(4.1)$ \\
\hline Hydralazine & $5(0.8)$ \\
\hline Miscellaneous $^{\mathrm{e}}$ & $9(1.5)$ \\
\hline Antidysrhythmics and other cardiovascular agents & $38(6.4)$ \\
\hline Atorvastatin & $8(1.3)$ \\
\hline Amiodarone & $7(1.2)$ \\
\hline Cardiovascular agent unspecified & $5(0.8)$ \\
\hline Sotalol & $5(0.8)$ \\
\hline Miscellaneous $^{\mathrm{f}}$ & $13(2.2)$ \\
\hline Cardiac glycosides & $30(5.0)$ \\
\hline Digoxin & $28(4.7)$ \\
\hline Digitoxin & $2(0.3)$ \\
\hline Diuretics & $29(4.9)$ \\
\hline Hydrochlorothiazide & $14(2.3)$ \\
\hline Furosemide & $8(1.3)$ \\
\hline Spironolactone & $5(0.8)$ \\
\hline Miscellaneous $^{\mathrm{g}}$ & $<5(<0.8)$ \\
\hline Angiotensin receptor blockers & $9(1.5)$ \\
\hline Losartan & $6(1.0)$ \\
\hline Miscellaneous $^{\mathrm{h}}$ & $<5(<0.8)$ \\
\hline Class total & $597(100)$ \\
\hline
\end{tabular}

${ }^{a}$ Includes dexmetetomidine and methyldopa

${ }^{\mathrm{b}}$ Includes nadolol

${ }^{\mathrm{c}}$ Includes felodipine and lercanidipine

${ }^{\mathrm{d}}$ Includes benazepril and quinipril

${ }^{\mathrm{e}}$ Includes nitroglycerin, doxazosin, isosorbide, tamsulosin, and terazosin

${ }^{\mathrm{f}}$ Includes flecainide, gemfibrozil, midodrine, simvastatin, disopyramide, ivabradine, lovastatin, and rosuvastatin

${ }^{\mathrm{g}}$ Includes chlorthalidone and pamabrom

${ }^{\mathrm{h}}$ Includes valsartan

reported with less than 5 cases. Caffeine was again the most common agent (25.6\%), followed by melatonin (21.4\%). Unspecified herbals/dietary supplements/vitamins were the next most common (9.4\%).
Table 18 Antipsychotics

\begin{tabular}{lc}
\hline & $N(\%)$ \\
\hline Quetiapine & $264(47.5)$ \\
Olanzapine & $81(14.6)$ \\
Risperidone & $40(7.2)$ \\
Aripiprazole & $39(7.0)$ \\
Haloperidol & $31(5.6)$ \\
Chlorpromazine & $19(3.4)$ \\
Clozapine & $19(3.4)$ \\
Ziprasidone & $17(3.1)$ \\
Lurasidone & $13(2.3)$ \\
Antipsychotic unspecified & $7(1.3)$ \\
Paliperidone & $5(0.9)$ \\
Miscellaneous & a \\
Class total & $21(3.8)$ \\
\hline
\end{tabular}

${ }^{\mathrm{a}}$ Includes asenapine, brexpiprazole, fluphenazine, loxapine, perphenazine, prochlorperazine, thiothixene, and trifluoperazine

\section{Gases, Irritants, Vapors, and Dusts}

Table 26 shows the gases, irritants, vapors, and dusts category. Carbon monoxide was the most commonly reported agent (53.2\%), consistent with prior years [5-8]. Chlorine and natural gas were reported with equal frequency (4.3\%). In 2017, a number of infrequently reported miscellaneous compounds made up a large portion of the agent class (35.1\%).

\section{Household Agents}

Table 27 presents the household product agent class. Cleaning solutions and disinfectants were most commonly reported (25.0\%), followed by sodium hypochlorite at a concentration less than or equal to $6 \%(19.1 \%)$. Laundry detergent pod exposures decreased slightly, making up $11.8 \%$ of the class in 2017 as compared to $13.3 \%$ in 2016.

\section{Plants and Fungi}

Table 28 summarizes the plants and fungi agent class. There were 65 agents entered with unspecified mold species being the predominant agent in the category (44.6\%). Other or unknown mushroom species was the next most common entry (12.3\%). Kratom (Mitragyna speciosa) entries remained stable from 2016 (9.2\%) [8]. Infrequent miscellaneous agents made up $26.2 \%$ of the class.

\section{Supplemental Tables}

Tables S1-S16 can be found in the Supplemental Materials. They present the less frequently reported agent classes, or those agent classes with little diversity, such as fewer than five 
Table 19 Anticonvulsants and mood stabilizers, and lithium

\begin{tabular}{lc}
\hline & $N(\%)$ \\
\hline Lithium $^{\mathrm{a}}$ & $121(100)$ \\
Lamotrigine & $108(27.1)$ \\
Valproic acid & $91(22.8)$ \\
Carbamazepine & $38(9.5)$ \\
Topiramate & $38(9.5)$ \\
Oxcarbazepine & $31(7.8)$ \\
Phenytoin & $30(7.5)$ \\
Levetiracetam & $24(6.0)$ \\
Divalproex & $10(2.5)$ \\
Lacosamide & $9(2.3)$ \\
Zonisamide & $5(1.3)$ \\
Miscellaneous & $15(3.8)$ \\
Class total & $399(100)$ \\
\hline
\end{tabular}

${ }^{a}$ Lithium is considered a separate agent class

${ }^{\mathrm{b}}$ Includes anticonvulsant unspecified, clobazam, felbamate, fosphenytoin, perampanel, primidone, rufinamide, and tiagabine

agent types, or where one agent made up a vast majority of the class. They are briefly described below.

\section{Cough and Cold Preparations}

Table S1 presents the 112 cough and cold product agent entries. As in prior years, dextromethorphan made up the majority of the category ( $84.8 \%)$. Unspecified cough and cold products were the next most common $(8.0 \%)$.

Table 20 Envenomations and marine poisonings

\begin{tabular}{ll}
\hline & $N(\%)$ \\
\hline Agkistrodon spp. & $108(28.8)$ \\
Crotalus spp. & $95(25.3)$ \\
Snake unspecified & $38(10.1)$ \\
Loxosceles spp. & $32(8.5)$ \\
Trimeresurus spp. (Asian pit vipers) & $25(6.7)$ \\
Chilopoda spp. (centipedes) & $15(4.0)$ \\
Envenomation unspecified & $14(3.7)$ \\
Latrodectus spp. & $10(2.7)$ \\
Micrurus spp. & $9(2.4)$ \\
Hymenoptera & $7(1.9)$ \\
Miscellaneous & \\
Class total & $19(5.1)$ \\
\end{tabular}

${ }^{a}$ Includes Centruroides spp., Vipera palaestinae, Homalopsis spp. (water snakes), Naja kaouthia, spider unspecified, Acanthanter plasti (Crown of Thorns starfish), animal bite unspecified, ciguetera poisoning, marine toxin unspecified, Micruroides spp., Scolopendra spp. (giant centipedes), scombroid poisoning, scorpion unspecified, and stingrays
Table 21 Psychoactives

\begin{tabular}{ll}
\hline & $N(\%)$ \\
\hline Molly_amphetamine-like hallucinogen ${ }^{\mathrm{a}}$ & $12(100)$ \\
Marijuana & $73(32.7)$ \\
Cannabinoid synthetic & $49(22.0)$ \\
LSD & $15(6.7)$ \\
Cannabinoid non-synthetic & $14(6.3)$ \\
Phencyclidine & $11(4.9)$ \\
Ketamine & $10(4.5)$ \\
Gamma hydroxybutyrate & $9(4.0)$ \\
Methylenedioxymethamphetamine & $9(4.0)$ \\
Delta-9-tetrahydrocannabinol & $8(3.6)$ \\
Miscellaneous & $25(11.2)$ \\
Class total & $223(100)$ \\
\hline
\end{tabular}

LSD lysergic acid diethylamide

${ }^{a}$ Amphetamine-like hallucinogens are considered a separate agent class

${ }^{\mathrm{b}}$ Includes 1,4-Butanediol, 3-methoxyphencyclidine, cannabidiol, disulfiram, donepezil, ethylone, gamma butyrolactone, hallucinogen unspecified, Ipracetin (4-acetoxy-DiPT, 4-acetoxy-N,N-diisopropyltryptamine), nicotine, pharmaceutical tetrahydrocannabinol (THC), psychoactive unspecified, and thujone

\section{Caustics}

Table S2 details the caustics agent class. In 2017, cleaning agents and formaldehyde were the most frequently reported agents $(10.1 \%)$. Miscellaneous caustics, each reported 4 or fewer times, made up more than one-third of the agent class $(36.0 \%)$.

\section{Hydrocarbons}

Table S3 presents the hydrocarbons agent class. Unspecified hydrocarbons were the most frequently reported (21.3\%), similar to prior years. In 2017, lamp oil and petroleum distillates each made up $8 \%$ of the agent class, an increase from the past few years [6-8].

\section{Antimicrobials}

Table S4 presents the antimicrobial agent class. The class is subdivided into antibiotics, antivirals, and other antimicrobial agents. In 2017, the top antibiotic agents were again amoxicillin (10.0\%) and dapsone (8.3\%), with isoniazid (5.0\%) and levofloxacin $(5.0 \%)$ following. Among the other antimicrobials, quinine was the most commonly reported (10.0\%). The antiviral subclass made up $16.7 \%$ of the agent class with amantadine $(5.0 \%)$ being the most common agent. 


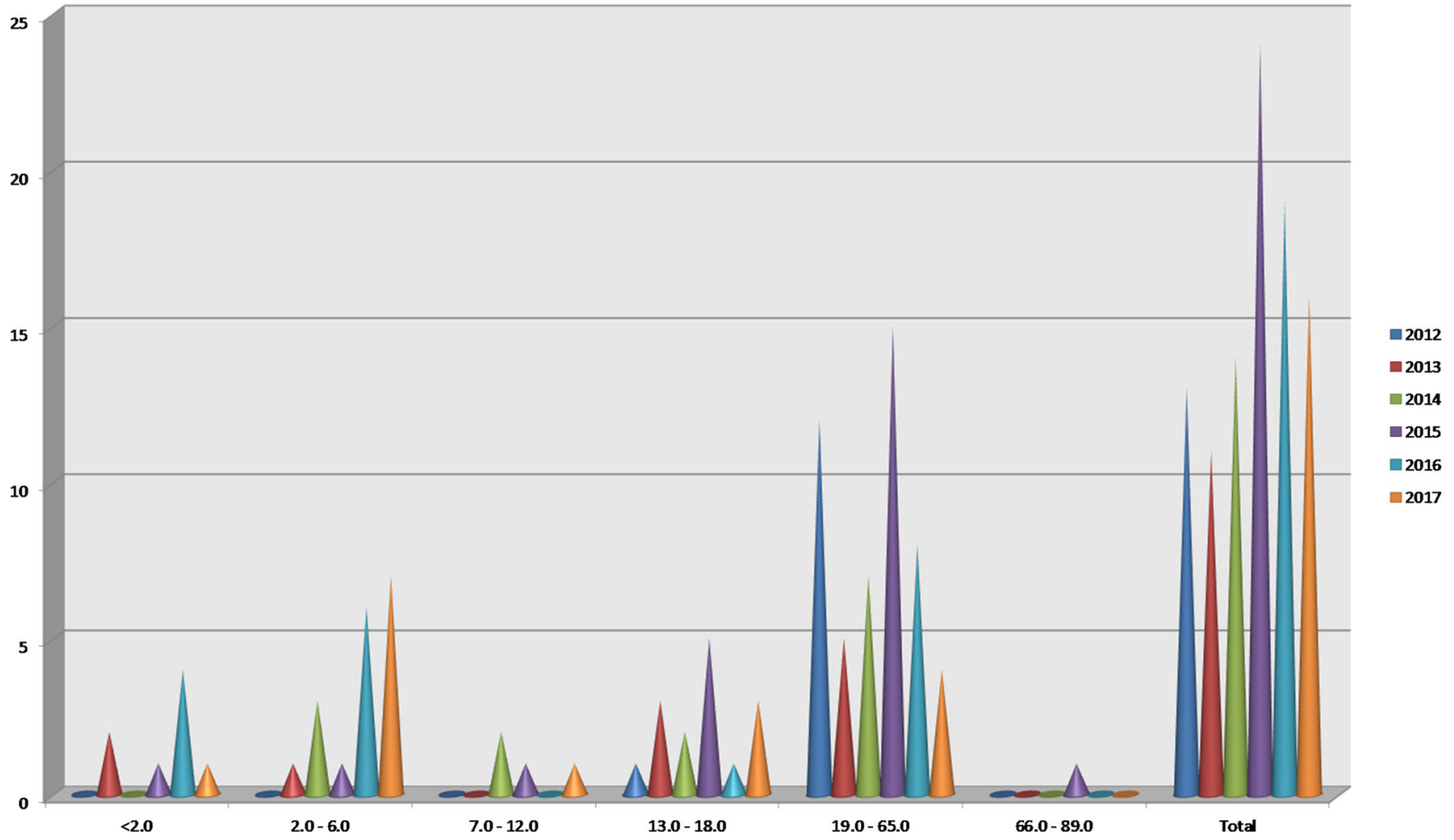

Fig. 2 Marijuana edibles from 2012 to 2017 by age group

\section{Endocrine}

Table S5 summarizes the 38 endocrine agents. Levothyroxine was the most commonly reported agent, making up $31.6 \%$ of the agent class. Prednisone was the next most commonly reported $(15.8 \%)$.

\section{Chemotherapeutic and Immunological Agents}

Table S6 summarizes the 35 chemotherapeutic and immunological agents. Methotrexate was again the most commonly reported agent $(22.9 \%)$. The majority of the class $(54.3 \%)$ was made up of infrequently reported miscellaneous agents.

\section{Other Non-pharmaceuticals}

Table S7 presents the 38 other non-pharmaceutical agents. Perfluoro and polyfluoroalkyl substances (PFASs) were the most commonly reported (18.2\%). Miscellaneous agents made up $51.5 \%$ of the agent class.

\section{Gastrointestinal Agents}

Table S 8 presents the 30 gastrointestinal agents. Omeprazole $(30.0 \%)$ and ondansetron $(10.0 \%)$ were the most commonly reported specific agents. Miscellaneous agents made up $60.0 \%$ of the class.

\section{Anesthetics}

Table S9 shows the anesthetic agent class. There were 27 anesthetic agents reported with almost half of the class $(48.1 \%)$ being made up of miscellaneous items. Lidocaine was the most commonly reported agent (29.6\%).

\section{Insecticides, Herbicides, Rodenticides, and Fungicides}

Table S10 presents the insecticide, rodenticide, and herbicide agent classes. There were no fungicide agents reported in 2017. There were 27 insecticide agent entries. Unspecified pyrethroids were the most commonly reported agents, making up $22.2 \%$ of the class. Miscellaneous agents made up $33.3 \%$ of the class. Rodenticides and herbicides were infrequently reported.

\section{Anticoagulants}

Table S11 summarizes the 24 entries in the anticoagulant class. Warfarin was again the most commonly reported agent and made up the majority of the class $(58.3 \%)$.

\section{Other Pharmaceuticals}

Table S12 presents the other pharmaceutical agent class. There were 18 entries in this category, with no agent contributing more than 3 cases. Hydrogen peroxide less than or equal to 
Table 22 Pediatric exposures to drugs of abuse

\begin{tabular}{|c|c|c|c|c|}
\hline \multicolumn{5}{|l|}{ A. Ethnicity } \\
\hline Ethnicity & \multicolumn{2}{|c|}{ Total number of pediatric patients } & \multicolumn{2}{|l|}{$\%$ of total } \\
\hline Hispanic & \multicolumn{2}{|c|}{20} & \multicolumn{2}{|l|}{16.2} \\
\hline Non-Hispanic & \multicolumn{2}{|l|}{50} & \multicolumn{2}{|l|}{40.6} \\
\hline Unknown & \multicolumn{2}{|l|}{53} & \multicolumn{2}{|l|}{43.1} \\
\hline \multicolumn{5}{|l|}{ B. Ethnicity by gender } \\
\hline Ethnicity by gender & Male & $\%$ of males & Female & $\%$ of females \\
\hline Hispanic & 15 & 22.4 & 5 & 8.9 \\
\hline Non-Hispanic & 45 & 67.2 & 5 & 8.9 \\
\hline Unknown & 7 & 10.4 & 46 & 82.1 \\
\hline \multicolumn{5}{|l|}{ C. Race } \\
\hline Race & \multicolumn{2}{|c|}{ Total \# of pediatric patients } & \multicolumn{2}{|l|}{$\%$ of total } \\
\hline Caucasian & \multicolumn{2}{|c|}{73} & 59.3 & \\
\hline African American & 26 & & 21.1 & \\
\hline Asian & 2 & & 1.6 & \\
\hline American Indian/Alaska Native & 1 & & 0.8 & \\
\hline Mixed & 2 & & 1.6 & \\
\hline Unknown & 19 & & 15 & \\
\hline D. Race by gender & & & & \\
\hline Race by Gender & Male & $\%$ of males & Female & $\%$ of female \\
\hline Caucasian & 37 & 55.2 & 36 & 64.3 \\
\hline African American & 13 & 19.4 & 13 & 23.2 \\
\hline Asian & 2 & 3.0 & 0 & 0 \\
\hline American Indian/Alaska Native & 0 & 0 & 1 & 1.8 \\
\hline Mixed & 2 & 3.0 & 0 & 0 \\
\hline Unknown & 13 & 19.4 & 6 & 10.7 \\
\hline E. Single and multiple ingestions & & & & \\
\hline Single vs multiple ingestion & & tric patients & & \\
\hline Single agent & 76 & & 61.8 & \\
\hline Multiple agents & 47 & & 38.2 & \\
\hline F. Single and multiple ingestions by $g$ & & & & \\
\hline Single vs multiple agents & Mal & $\%$ of males & Females & $\%$ of females \\
\hline Single & 44 & 65.7 & 32 & 57.1 \\
\hline Multiple & 23 & 34.3 & 24 & 42.9 \\
\hline $\begin{array}{l}\text { G. Agent classes involved in pediatric } \\
\text { drugs of abuse }\end{array}$ & & & & \\
\hline Agent class & & tric patients & & \\
\hline Psychoactive & 57 & & 46.3 & \\
\hline Sympathomimetic & 54 & & 43.9 & \\
\hline Opioid & 29 & & 23.6 & \\
\hline Antidepressant & 10 & & 8.1 & \\
\hline Sedative-hypnotic/muscle relaxant & 7 & & 5.7 & \\
\hline Anticholinergic/antihistamine & 4 & & 3.3 & \\
\hline Amphetamine-like hallucinogen & 4 & & 3.3 & \\
\hline Antipsychotic & 4 & & 3.3 & \\
\hline Herbals/dietary supplements & 3 & & 2.4 & \\
\hline Analgesics & 3 & & 2.4 & \\
\hline Cardiovascular & 2 & & 2.4 & \\
\hline Alcohol-ethanol & 2 & & 2.4 & \\
\hline $\begin{array}{l}\text { H. Agent classes involved in pediatric } \\
\text { drugs of abuse by gender }\end{array}$ & & & & \\
\hline Agent class & Males & $\%$ of males & Females & $\%$ of females \\
\hline Psychoactive & 32 & 47.7 & 25 & 44.6 \\
\hline Sympathomimetic & 30 & 44.8 & 24 & 42.8 \\
\hline Opioid & 16 & 23.9 & 13 & 23.2 \\
\hline Antidepressant & 3 & 4.5 & 1 & 1.8 \\
\hline Sedative-hypnotic/muscle relaxant & 3 & 4.5 & 4 & 7.1 \\
\hline Anticholinergic/antihistamine & 3 & 4.5 & 1 & 1.8 \\
\hline Antipsychotic & 2 & 2.9 & 2 & 3.6 \\
\hline Herbals/dietary supplements & 2 & 2.9 & 1 & 1.8 \\
\hline Analgesics & 0 & 0 & 3 & 5.4 \\
\hline Cardiovascular & 1 & 1.5 & 4 & 7.1 \\
\hline Alcohol-ethanol & 1 & 1.5 & 1 & 1.8 \\
\hline I. Reason for medical toxicology enco & & & & \\
\hline Reason for encounter & & tric patients & & \\
\hline Intentional pharmaceutical & 34 & & 27.6 & \\
\hline Attempt at self-harm & 16 & & 13.0 & \\
\hline
\end{tabular}


Table 22 (continued)

\begin{tabular}{|c|c|c|c|c|}
\hline Misuse/abuse & 12 & & 9.8 & \\
\hline Therapeutic use & 2 & & 1.6 & \\
\hline Unknown & 4 & & 3.3 & \\
\hline Intentional non-pharmaceutical & 47 & & 38.2 & \\
\hline Attempt at self-harm & 1 & & 0.8 & \\
\hline Misuse/abuse & 26 & & 21.1 & \\
\hline Use for therapeutic intent & 1 & & 0.8 & \\
\hline Drug concealment & 1 & & 0.8 & \\
\hline Unknown & 18 & & 14.6 & \\
\hline Unintentional pharmaceutical & 13 & & 10.6 & \\
\hline Unintentional non- & 27 & & 21.9 & \\
\hline \multicolumn{5}{|l|}{ pharmaceutical } \\
\hline Malicious/criminal & 1 & & 0.8 & \\
\hline Interpretation of tox data & 1 & & 0.8 & \\
\hline \multicolumn{5}{|c|}{$\begin{array}{l}\text { J. Reason for medical toxicology encounter by } \\
\text { gender }\end{array}$} \\
\hline Reason for encounter by gender & Male & $\%$ of males & Female & $\%$ of female \\
\hline Intentional pharmaceutical & 20 & 29.8 & 14 & 25.0 \\
\hline Attempt at self-harm & 8 & 11.9 & 8 & 14.3 \\
\hline Misuse/abuse & 9 & 13.4 & 3 & 5.4 \\
\hline Therapeutic use & 1 & 1.5 & 1 & 1.8 \\
\hline Unknown & 2 & 2.9 & 2 & 3.6 \\
\hline Intentional non-pharmaceutical & 28 & 41.8 & 19 & 33.9 \\
\hline Attempt at self-harm & 0 & 0 & 1 & 1.8 \\
\hline Misuse/Abuse & 24 & 35.8 & 2 & 3.6 \\
\hline Use for therapeutic intent & 1 & 1.5 & 0 & 0 \\
\hline Drug concealment & 1 & 1.5 & 0 & 0 \\
\hline Unknown & 2 & 2.98 & 16 & 28.6 \\
\hline Unintentional Pharmaceutical & 8 & 11.9 & 5 & 8.9 \\
\hline Unintentional non- & 10 & 14.9 & 17 & 30.3 \\
\hline \multicolumn{5}{|l|}{ pharmaceutical } \\
\hline Malicious/criminal & 1 & 1.5 & 0 & 0 \\
\hline Interpretation of tox data & 0 & 0 & 1 & 1.8 \\
\hline
\end{tabular}

$10 \%$ and sumatriptan were the most commonly reported, each accounting for $16.7 \%$ of the class.

\section{Weapons of Mass Destruction}

Table S13 summarizes the potential weapons of mass destruction/riot agents/ radiological agents class. There were 7 entries with $42.9 \%$ being botulinum toxin.

Table 23 Diabetic medications

\begin{tabular}{ll}
\hline & $N(\%)$ \\
\hline Metformin & $57(33.1)$ \\
Glipizide & $40(23.3)$ \\
Insulin & $38(22.1)$ \\
Glimepiride & $15(8.7)$ \\
Glyburide & $12(7.0)$ \\
Miscellaneous $^{\mathrm{a}}$ & $10(5.8)$ \\
Class total & $172(100)$
\end{tabular}

${ }^{a}$ Includes diabetic medication unspecified, sitagliptin, alogliptin, exenatide, and liraglutide

\section{Anti-Parkinsonism Agents}

Table S14 presents the 6 entries for the anti-parkinsonism agent class. Levodopa/carbidopa and pramipexole were reported in equal numbers each making up $33.3 \%$ of the agent class.

Table 24 Metals

\begin{tabular}{ll}
\hline & $N(\%)$ \\
\hline Lead & $32(27.8)$ \\
Iron & $26(22.6)$ \\
Mercury & $11(9.6)$ \\
Cobalt & $10(8.7)$ \\
Arsenic & $6(5.2)$ \\
Chromium & $6(5.2)$ \\
Miscellaneous & a \\
Class total & $24(20.9)$ \\
\hline
\end{tabular}

${ }^{\mathrm{a}}$ Includes magnesium, cadmium, copper, gadolinium, manganese, aluminum, antimony, beryllium, cesium, metal unspecified, selenium, titanium, and zinc 
Table 25 Herbal products and dietary supplements

\begin{tabular}{ll}
\hline & $N(\%)$ \\
\hline Caffeine & $30(25.6)$ \\
Melatonin & $25(21.4)$ \\
Herbals/dietary supplements/vitamins unspecified & $17(14.5)$ \\
Multiple vitamin & $5(4.3)$ \\
Vitamin D & $5(4.3)$ \\
Eucalyptus oil & $5(4.3)$ \\
Limonene & $3(2.6)$ \\
Miscellaneous & a \\
Class total & $27(23.1)$ \\
\end{tabular}

${ }^{\text {a }}$ Includes aloin (aloe vera extract or outer leaves), ashwangandha, black cohosh, Brazil seed (Bertholletia excelsa), citronella oil, dietary supplement unspecified, eugenol (clove oil), herbal (dietary) multibotanical, minerals unspecified, omega-3-acid ethyl esters, orange oil, potassium, senna, sodium chloride, tryptophan, vitamin A, vitamin B1 (thiamine), vitamin B3 (niacin), vitamin B6 (pyridoxine), vitamin C (ascorbic acid), and yohimbine

\section{Foreign Bodies}

Table S15 presents the ingested foreign bodies agent class. These were infrequently reported with 5 entries, 2 of which were batteries $(40.0 \%)$.

\section{Pulmonary Agents}

Table S16 reports the 4 pulmonary agent entries. Albuterol made up $50.0 \%$ of the category.

\section{Clinical Signs and Symptoms}

The various clinical signs and symptoms categories report information on a diverse range of abnormal clinical findings. In order to be reported as being present, it must meet predefined criteria. For example, tachycardia is defined as a heart

Table 26 Gases, irritants, vapors, and dusts

\begin{tabular}{ll}
\hline & $N(\%)$ \\
\hline Carbon monoxide & $50(53.2)$ \\
Chlorine & $4(4.3)$ \\
Natural gas & $4(4.3)$ \\
Hydrogen sulfide & $3(3.2)$ \\
Miscellaneous $^{\mathrm{a}}$ & $33(35.1)$ \\
Class total & $94(100)$ \\
\hline
\end{tabular}

${ }^{a}$ Includes acetonitrile, asbestos, bromine, carbon dioxide, chloramine, cyanide, diesel exhaust, dust, duster (canned air), fumes/vapors/gases unspecified, gases/vapors/irritants/dusts unspecified, halon, metal dust unspecified, nitric oxide, nitrogen oxides, ozone, petroleum vapors, polyurethane vapors, radon, smoke, sulfur dioxide, volatile organic compounds (VOC) unspecified, welding fumes, and wood dusts
Table 27 Household products

\begin{tabular}{lc}
\hline & $N(\%)$ \\
\hline Cleaning solutions and disinfectants & $17(25.0)$ \\
Sodium hypochlorite $\leq 6 \%$ & $13(19.1)$ \\
Soaps and detergents & $11(16.2)$ \\
Laundry detergent pod & $8(11.8)$ \\
Hair product & $4(5.9)$ \\
Paint & $4(5.9)$ \\
Miscellaneous & a \\
Class total & $11(16.2)$ \\
\hline${ }^{a}$ Includes brake fluid, dishwasher detergent, dishwasher detergent pod, \\
fabric softener, household product unspecified, phenylenediamine (hair \\
dye), rubber cement, and sunscreens
\end{tabular}

rate greater than 140 beats per minute. Additionally, each case may report more than one abnormality within a group or across groups. For example, a single case entry may have more than one vital sign abnormality, or may have both a vital sign abnormality and a neurological abnormality. The percentages for these categories are calculated relative to the total number of Registry cases. It is possible for the total to be more than $100 \%$.

\section{Toxidromes}

Table 29 summarizes the 2779 toxidrome entries in the Registry in 2017. The frequency of reported toxidromes remained consistent with prior years with the sedativehypnotic toxidrome being by far the most commonly reported (15.7\%). The anticholinergic toxidrome was the next most common $(7.3 \%)$, followed by sympathomimetic $(5.1 \%)$, opioid $(3.7 \%)$, and serotonin syndrome $(2.9 \%)$.

Table 28 Plants and fungi

\begin{tabular}{lc}
\hline & $N(\%)$ \\
\hline Mold unspecified & $29(44.6)$ \\
Mushroom, other/unknown & $8(12.3)$ \\
Mitragyna speciosa (kratom) & $6(9.2)$ \\
Mushroom, psilocibin $^{\text {Miscellaneous }}{ }^{2}$ & $5(7.7)$ \\
Class total & $17(26.2)$ \\
\hline
\end{tabular}

${ }^{a}$ Includes Aesculus hippocastanum (horse chestnut), Akuamma (Picralima nitida), Amanita phalloides, Datura stramonium (jimsonweed), Hydrastis canadensis (goldenseal), Lupinus (lupini beans), moonflower, Nerium oleander, strychnine, Toxicoscordion venenosum (death camas), Valerian root, and Vicia faba (fava bean) 


\section{Major Vital Sign Abnormalities}

Table 30 summarizes the 2001 recorded major vital sign abnormalities. This represents $26.4 \%$ of the Registry, though cases may be associated with more than one major vital sign abnormality. The vital sign abnormalities remained similar in number to prior years. Tachycardia was the most commonly reported $(11.8 \%)$. Hypotension was the next most common $(6.2 \%)$.

\section{Clinical Signs and Symptoms-Neurological}

Table 31 presents the 6138 entries recording neurological signs and symptoms (81.0\%). Coma/central nervous system depression remained the most commonly reported sign $(33.3 \%)$. Agitation (16.5\%) and delirium $(11.1 \%)$ were the next most common.

\section{Clinical Signs-Cardiovascular and Pulmonary}

Table 32 presents the cardiovascular and pulmonary clinical signs. The most frequently reported cardiovascular sign was prolonged QTc $(6.1 \%)$ followed by prolonged QRS $(1.8 \%)$ and myocardial injury or infarction (1.5\%). Among pulmonary signs, respiratory depression was the most commonly reported (10.4\%) and made up the majority of this category.

\section{Clinical Signs-Other Organ Systems}

Table 33 presents the other organ system clinical signs. Among these additional categories, metabolic abnormalities were most frequently reported $(12.4 \%)$. Cases reporting an elevated anion gap (5.2\%) and a metabolic acidosis $(4.7 \%)$ made up the majority of this category. The renal/ musculoskeletal category was the next most common and cases were fairly evenly distributed between rhabdomyolysis $(5.1 \%)$ and acute kidney injury $(4.1 \%)$. Hematological signs made up $6.7 \%$ of the other organ systems. Coagulopathy $(2.5 \%)$ and leukocytosis $(1.8 \%)$ were the most commonly reported hematological signs. Among the gastrointestinal/ hepatic signs, hepatotoxicity $(3.2 \%)$ was the most common, followed by gastrointestinal bleeding $(0.7 \%)$ and pancreatitis $(0.6 \%)$. Dermatological signs were the least commonly reported category $(4.5 \%)$. Rash $(2.1 \%)$ and blister/bullae $(1.1 \%)$ were the most common dermatological signs reported.

\section{Fatalities}

Tables 34 and 35 summarize cases which reported fatalities. Table 34 includes cases involving single agent exposures, and Table 35 presents those fatalities involving multiple agents. Table S17 in the Supplementary materials presents those fatalities in which it is unknown whether there was a related toxicological exposure.

There were a total of 93 fatalities in 2017, involving $1.2 \%$ of Registry cases. Forty-seven cases involved single agent exposures, 21 involved multiple agents, and 25 cases were unknown. The number of fatality cases decreased from 2016, though was similar to the years prior to that [6-8].

Seventeen fatality cases involved at least one opioid agent. Eight cases involved heroin. Two additional cases involved fentanyl or a fentanyl derivative, and 1 case involved U47700. There were 6 opioid fatalities in which only the single opioid agent was involved, the remainder were polypharmacy exposures, and some involved multiple opioids. Sedative-hypnotics/muscle relaxants, including gabapentinoids, were involved in 14 fatality cases. There were five cases that involved both a sedative-hypnotics/muscle relaxant and an opioid.

In 2017 , there were 10 pediatric (age 0-18 years) deaths related to toxicologic exposures. The age range for these was 11 months to 18 years. Six of these were single agent exposures and 4 involved multiple agents. Two deaths were single agent exposures to acetaminophen, both in 14 year olds. One death involved an 18 year-old with a bupropion exposure that resulted in hypotension, ventricular dysrhythmia, QRS prolongation, QTc prolongation, myocardial injury, respiratory depression, metabolic acidosis, and central nervous system depression. He was treated with sodium bicarbonate, lipid resuscitation, vasopressors, intubation, and cardiopulmonary resuscitation. There was a death in an 11 month old related to cocaine and methamphetamine who had clinical signs of hypotension, tachycardia, agitation, and reactive airway disease and was treated with benzodiazepines. A 16 year old died related to an unspecified opioid after life support was withdrawn.

There were 38 fatality cases in which life support was withdrawn ( $0.5 \%$ of Registry cases). An additional, 7 cases were unknown whether life support was withdrawn. Brain death was declared in 12 cases, though there were 19 cases in which it was unknown if brain death was declared.

\section{Adverse Drug Reactions}

Table 36 presents the drugs most frequently associated with adverse drug reactions (ADRs). Lithium was again the most commonly reported agent in 2017 (4.1\%), as it has been in past years. The next most commonly reported agentsvalproic acid (3.8\%), haloperidol (2.9\%), and bupropion $(2.7 \%)$ - have also been reported in past years. Digoxin was less frequently reported in 2017 and was not one of the top 10 agents as it has been in prior years [5-8]. The sedatives clonazepam $(2.4 \%)$ and baclofen $(2.1 \%)$ were among the most commonly reported agents associated with ADRs in 2017. This was a change from prior years, though in 2015, lorazepam was the second most commonly reported ADR [7]. 
Table 29 Toxidromes

\begin{tabular}{ll}
\hline & $N(\%)^{\mathrm{a}}$ \\
\hline Sedative-hypnotic & $1192(15.7)$ \\
Anticholinergic & $550(7.3)$ \\
Sympathomimetic & $387(5.1)$ \\
Opioid & $280(3.7)$ \\
Serotonin syndrome & $217(2.9)$ \\
Alcoholic ketoacidosis & $58(0.8)$ \\
Sympatholytic & $37(0.5)$ \\
NMS & $17(0.2)$ \\
Washout syndrome & $16(0.2)$ \\
Overlap syndromes (MCS, chronic fatigue, etc.) & $13(0.2)$ \\
Anticonvulsant hypersensitivity & $5(0.1)$ \\
Miscellaneous & $7(0.1)$ \\
Total & $2779(36.7)$ \\
\hline
\end{tabular}

NMS neuroleptic malignant syndrome

${ }^{a}$ Percentage equals number cases reporting specific toxidrome relative to total number of Registry cases in $2017(N=7577)$

${ }^{\mathrm{b}}$ Includes cholinergic, fume fever

\section{Treatment}

\section{Antidotal Therapy Administered}

Table 37 summarizes antidotal therapies reported to the Registry. Similar to prior years, $\mathrm{N}$-acetylcysteine was the most commonly reported antidote (28.3\%), followed by naloxone/ nalmefene $(21.9 \%)$ and sodium bicarbonate (11.8\%).

\section{Antivenom Therapy Administered}

Table 38 summarizes the antivenom therapies reported in 2017. Similar to prior years, Crotalidae polyvalent immune fab (ovine) was the most commonly reported antivenom

Table 30 Major vital sign abnormalities

\begin{tabular}{ll}
\hline & $N(\%)^{\mathrm{a}}$ \\
\hline Tachycardia $(\mathrm{HR}>140)$ & $896(11.8)$ \\
Hypotension (systolic $\mathrm{BP}<80 \mathrm{mmHg})$ & $471(6.2)$ \\
Bradycardia $(\mathrm{HR}<50)$ & $264(3.5)$ \\
Bradypnea $(\mathrm{RR}<10)$ & $205(2.7)$ \\
Hypertension $($ systolic $\mathrm{BP}>200 \mathrm{mmHg}$ and/or diastolic & $123(1.6)$ \\
$\quad$ BP $>120 \mathrm{mmHg})$ & $42(0.6)$ \\
Hyperthermia (temp $\left.>105{ }^{\circ} \mathrm{F}\right)$ & $2001(26.4)^{\mathrm{b}}$ \\
Total &
\end{tabular}

$H R$ heart rate, $B P$ blood pressure, $R R$ respiratory rate

${ }^{a}$ Percentage equals the number of cases relative to the total number of Registry cases in $2017(N=7577)$

${ }^{\mathrm{b}}$ Total reflects cases reporting at least one major vital sign abnormality. Cases may be associated with more than one major vital sign abnormality
Table 31 Clinical signs and symptoms - neurological

\begin{tabular}{ll}
\hline & $N(\%)^{\mathrm{a}}$ \\
\hline Coma/CNS depression & $2524(33.3)$ \\
Agitation & $1251(16.5)$ \\
Delirium & $843(11.1)$ \\
Hyperreflexia/myoclonus/tremor & $480(6.3)$ \\
Seizures & $427(5.6)$ \\
Hallucinations & $312(4.1)$ \\
Dystonia/rigidity/extrapyramidal symptoms & $106(1.4)$ \\
Numbness/paresthesia & $93(1.2)$ \\
Weakness/paralysis & $76(1.0)$ \\
Peripheral neuropathy & $26(0.3)$ \\
Total & $6138(81.0)^{\mathrm{a}, \mathrm{b}}$ \\
\hline
\end{tabular}

CNS central nervous system

${ }^{\text {a }}$ Percentage equals number cases relative to total number of Registry cases in $2017(N=7577)$

${ }^{\mathrm{b}}$ Total reflects cases reporting at least one neurological symptom. Cases may be associated with more than one neurological symptom

$(92.0 \%)$. Other snake venoms were the next most common and made up $3.7 \%$ of the category.

\section{Pharmaceutical Supportive Care}

Table 39 presents the pharmacological supportive care interventions that were reported in 2017. There were 3574 pharmacological interventions recorded. As in past years, benzodiazepines made up approximately half of the reported

Table 32 Clinical signs - cardiovascular and pulmonary

\begin{tabular}{ll}
\hline & $N(\%)^{\mathrm{a}}$ \\
\hline Cardiovascular & \\
Prolonged QTc $(\geq 500 \mathrm{~ms})$ & $460(6.1)$ \\
Prolonged QRS $(\geq 120 \mathrm{~ms})$ & $138(1.8)$ \\
Myocardial injury or infarction & $115(1.5)$ \\
Ventricular dysrhythmia & $71(0.9)$ \\
AV Block (>1st degree) & $29(0.4)$ \\
Total & $813(10.7)^{\mathrm{b}}$ \\
Pulmonary & \\
Respiratory depression & $785(10.4)$ \\
Aspiration pneumonitis & $197(2.6)$ \\
Acute lung injury/ARDS & $110(1.5)$ \\
Asthma/reactive airway disease & $49(0.6)$ \\
Total & $1141(15.1)^{\mathrm{b}}$ \\
\hline
\end{tabular}

$A R D S$ acute respiratory distress syndrome

${ }^{\text {a }}$ Percentage equals number cases reporting signs of symptoms relative to total number of Registry cases in $2017(\mathrm{~N}=7577)$

${ }^{\mathrm{b}}$ Total reflects cases reporting at least one cardiovascular or pulmonary symptom. Cases may be associated with more than one symptom 
Table 33 Clinical signs - other organ systems

\begin{tabular}{ll}
\hline & $N(\%)^{\mathrm{a}}$ \\
\hline Metabolic & \\
Elevated anion gap $(>20)$ & $394(5.2)$ \\
Metabolic acidosis $(\mathrm{pH}<7.2)$ & $357(4.7)$ \\
Hypoglycemia $($ glucose $<50 \mathrm{mg} / \mathrm{dL})$ & $131(1.7)$ \\
Elevated osmole gap $(>20)$ & $60(0.8)$ \\
Total & $942(12.4)^{\mathrm{b}}$ \\
Renal/Musculoskeletal & \\
Rhabdomyolysis $(\mathrm{CPK}>1000 \mathrm{IU} / \mathrm{L})$ & $383(5.1)$ \\
Acute kidney injury $(\mathrm{creatinine}>2.0 \mathrm{mg} / \mathrm{dL})$ & $309(4.1)$ \\
Total & $692(9.1)^{\mathrm{b}}$ \\
Hematological & \\
Coagulopathy $(\mathrm{PT}>15 \mathrm{~s})$ & $188(2.5)$ \\
Leukocytosis $(\mathrm{WBC}>20 \mathrm{~K} / \mu \mathrm{L})$ & $139(1.8)$ \\
Thrombocytopenia $(\mathrm{platelets}<100 \mathrm{~K} / \mu \mathrm{L})$ & $83(1.1)$ \\
Hemolysis $(\mathrm{Hgb}<10 \mathrm{~g} / \mathrm{dL})$ & $62(0.8)$ \\
Methemoglobinemia $(\mathrm{MetHgb} \geq 2 \%)$ & $21(0.3)$ \\
Pancytopenia & $16(0.2)$ \\
Total & $509(6.7)^{\mathrm{b}}$ \\
Gastrointestinal/hepatic & \\
Hepatotoxicity $(\mathrm{AST} \geq 1000 \mathrm{IU} / \mathrm{L})$ & $243(3.2)$ \\
Gastrointestinal bleeding & $54(0.7)$ \\
Pancreatitis & $45(0.6)$ \\
Corrosive injury & $342(4.5)^{\mathrm{b}}$ \\
Intestinal ischemia & $36(0.7)$ \\
Total & $34(0.4)$ \\
Dermatological & $380(5.0)^{\mathrm{b}}$ \\
Rash & \\
Blister/bullae & \\
Angioedema & \\
Necrosis & \\
Total & \\
\hline
\end{tabular}

AST aspartate aminotransferase, $P T$ prothrombin time, $W B C$ white blood cells, $H g b$ hemoglobin, $C P K$ creatine phosphokinase

${ }^{a}$ Percentage equals the number of cases reporting specific clinical signs compared to the total number of Registry cases in $2017(N=7577)$

${ }^{\mathrm{b}}$ Total reflects cases reporting at least one sign in the category. Cases may be associated with more than one symptom

pharmacologic interventions (49.1\%). Opioids (14.3\%) and vasopressors $(8.4 \%)$ were the next most commonly reported. There were 2501 cases $(33.0 \%)$ that involved at least one form of pharmacological supportive care.

\section{Non-pharmaceutical Supportive Care}

Table 40 presents the non-pharmacological supportive care interventions. The majority of this category was made up of intravenous fluid resuscitation $(72.3 \%)$. Intubation/ventilatory management was the next most common intervention
$(22.9 \%)$. Additional interventions were less common with none making up more than $2 \%$ of the category. Overall, 3141 Registry cases $(41.5 \%)$ reported at least one nonpharmacological intervention.

\section{Chelation Therapy Administered}

Table 41 summarizes chelation therapy reported to the Registry in 2017. There were 31 total chelator agents reported in 2017. Twenty-five cases involved the delivery of a single chelating agent, while 5 cases involved two or more agents. The most commonly administered chelator was dimercaptosuccinic acid (DMSA) (38.7\%). Deferoxamine was the next most common $(25.8 \%)$.

\section{Decontamination Interventions Administered}

Table 42 presents decontamination interventions. Activated charcoal remained by a large margin the most commonly reported decontamination measure $(79.6 \%)$. External irrigation $(8.1 \%)$ and whole bowel irrigation $(7.7 \%)$ were the next most commonly reported. Gastric lavage was an uncommon intervention (4.6\%). Overall, there were 264 cases that reported at least one kind of intervention (3.5\%). Some cases reported more than one decontamination method.

\section{Enhanced Elimination Interventions Administered}

Table 43 describes enhanced elimination techniques reported to the Registry. Urinary alkalinization was the most commonly reported intervention (26.4\%). Hemodialysis for toxin removal was the next most common (24.8\%) and hemodialysis for any other indication made up an additional $18.6 \%$ of the treatments in this group. Continuous renal replacement therapy made up $24.8 \%$ of the enhanced elimination techniques. There were 226 cases (3.0\% of the Registry) reporting at least one enhanced elimination method. Some cases reported more than one.

Table 44 presents more detailed information about cases in which extracorporeal membrane oxygenation ECMO was delivered from 2013 through 2017. During the 5-year period of data collection, there were 62 cases reporting the use of ECMO. The mean age of patients was 22 years. Most cases involved intentional ingestions.

\section{Discussion}

The data collection in this eighth year of the ToxIC case registry has been characterized by continued strong data collection and a number of important observations related to new trends and findings. Although the slight reduction in the 
Table 342017 fatalities reported in ToxIC Registry with known toxicological exposure: single agent

\begin{tabular}{|c|c|c|c|c|c|}
\hline $\begin{array}{l}\text { Age/ } \\
\text { gender }^{\mathrm{a}}\end{array}$ & Agents involved & Clinical findings & $\begin{array}{l}\text { Life } \\
\text { support } \\
\text { withdrawn }\end{array}$ & $\begin{array}{l}\text { Brain } \\
\text { death } \\
\text { confirmed }\end{array}$ & Treatment $^{\mathrm{b}}$ \\
\hline $51 \mathrm{~F}$ & Acetaminophen & $\begin{array}{l}\text { ALI, AGT, CNS, DLM, RFX, } \\
\text { MA, AG, HPT, HYS, GIB, } \\
\text { PLT, AKI }\end{array}$ & Yes & Unknown & $\begin{array}{l}\text { NAC, vasopressors, continuous renal replacement, intubation, IV } \\
\text { fluids }\end{array}$ \\
\hline $14 \mathrm{~F}$ & Acetaminophen & None listed & No & No & $\mathrm{NAC}$ \\
\hline $34 \mathrm{~F}$ & Acetaminophen & $\begin{array}{l}\text { HTN, TC, RD, CNS, MA, HPT, } \\
\text { CPT, AKI }\end{array}$ & No & No & $\begin{array}{l}\text { NAC, vitamin K, vasopressors, continuous renal replacement, } \\
\text { intubation }\end{array}$ \\
\hline $35 \mathrm{M}$ & Acetaminophen & HT, TC, CNS, MA, HPT, CPT & No & No & NAC, vitamin K, continuous renal replacement, intubation \\
\hline $53 \mathrm{~F}$ & Acetaminophen & CNS, MA, HPT, CPT, AKI & Yes & No & $\begin{array}{l}\text { NAC, vasopressors, corticosteroids, continuous renal replacement, } \\
\text { intubation }\end{array}$ \\
\hline $37 \mathrm{M}$ & Acetaminophen & $\begin{array}{l}\text { HT, TC, AP, CNS, DLM, MA, } \\
\text { AG, HPT, GIB, CPT, WBC, } \\
\text { AKI }\end{array}$ & Yes & Unknown & NAC, hemodialysis, intubation, IV fluids \\
\hline $38 \mathrm{M}$ & Acetaminophen & HPT, OTH2 & Yes & No & $\begin{array}{l}\text { NAC, thiamine, vasopressors, benzodiazepines, neuromuscular } \\
\text { blockers, opioids, continuous renal replacement, intubation, IV } \\
\text { fluids, transfusion }\end{array}$ \\
\hline $14 \mathrm{~F}$ & Acetaminophen & HPT & No & No & $\mathrm{NAC}$ \\
\hline $55 \mathrm{~F}$ & Amitriptyline & $\begin{array}{l}\text { HT, QRS, QTC, RD, CNS, SZ, } \\
\text { AKI }\end{array}$ & No & No & $\mathrm{NaHCO}_{3}$, benzodiazepines, intubation, IV fluids \\
\hline $52 \mathrm{~F}$ & Amlodipine & $\mathrm{HT}, \mathrm{BC}, \mathrm{RD}, \mathrm{CNS}, \mathrm{MA}$ & No & No & $\begin{array}{l}\text { Calcium, glucagon, insulin-euglycemic therapy, methylene blue, } \\
\text { vasopressors, neuromuscular blockers, opioids, continuous renal } \\
\text { replacement, CPR, intubation, IV fluids }\end{array}$ \\
\hline $60 \mathrm{M}$ & Amphetamine & $\begin{array}{l}\text { MI, ALI, AGT, DLM, RFX, MA, } \\
\text { AG, HPT, PLT, AKI, RBM }\end{array}$ & Yes & No & $\begin{array}{l}\text { Fomepizole, NAC, vasopressors, bronchodilators, antiarrhythmics, } \\
\text { benzodiazepines, neuromuscular blockers, opioids, continuous } \\
\text { renal replacement, CPR, aortic balloon pump }\end{array}$ \\
\hline $18 \mathrm{M}$ & Bupropion & $\begin{array}{l}\text { HT, VD, QRS, QTC, MI, RD, } \\
\text { CNS, MA }\end{array}$ & Yes & Yes & $\begin{array}{l}\text { Lipid resuscitation, } \mathrm{NaHCO}_{3} \text {, vasopressors, } \mathrm{CPR} \text {, intubation, IV } \\
\text { fluids }\end{array}$ \\
\hline $71 \mathrm{M}$ & Carbon monoxide & ALI, CNS, AG & No & No & Hydroxocobalamin \\
\hline $48 \mathrm{~F}$ & Carbon monoxide & HT, QTC, MI, RD, CNS, MA & No & No & Vasopressors, CPR, intubation \\
\hline $33 \mathrm{~F}$ & Clonazepam & CNS & No & No & None listed \\
\hline $47 \mathrm{M}$ & Cocaine & $\begin{array}{l}\text { HT, HYT, VD, CNS, MA, GIB, } \\
\text { AKI }\end{array}$ & Unknown & Unknown & $\begin{array}{l}\text { Vasopressors, activated charcoal, hemodialysis, continuous renal } \\
\text { replacement, CPR, intubation, IV fluids }\end{array}$ \\
\hline $43 \mathrm{~F}$ & Cocaine & CNS, MA, AG, HPT, AKI, RBM & No & No & $\begin{array}{l}\text { Calcium, lipid resuscitation, naloxone, } \mathrm{NaHCO}_{3} \text {, intubation, IV } \\
\text { fluids }\end{array}$ \\
\hline $51 \mathrm{M}$ & Cocaine & $\begin{array}{l}\text { MI, RD, AGT, DLM, SZ, WBC, } \\
\text { RBM }\end{array}$ & Yes & No & Vasopressors, antihypertensives, CPR, intubation, IV fluids \\
\hline $65 \mathrm{~F}$ & Colchicine & $\begin{array}{l}\text { HT, BC, MI, CNS, MA, PCT, } \\
\text { AKI, OTH2 }\end{array}$ & No & No & None listed \\
\hline $23 \mathrm{M}$ & Digitoxin & $\mathrm{HT}, \mathrm{BC}, \mathrm{VD}, \mathrm{QRS}, \mathrm{MA}, \mathrm{AG}, \mathrm{AKI}$ & Yes & No & Digoxin Fab \\
\hline $18 \mathrm{~F}$ & Diphenhydramine & AGT & No & No & Physostigmine, benzodiazepines, IV fluids \\
\hline $19 \mathrm{M}$ & Diphenhydramine & AGT, DLM & No & No & Benzodiazepines, IV fluids \\
\hline $47 \mathrm{~F}$ & Doxepin & $\begin{array}{l}\text { HT, TC, BP, HYT, VD, QRS, MI, } \\
\text { RD, CNS, MA, HYS, CPT, } \\
\text { WBC, AKI, RBM }\end{array}$ & Yes & Unknown & $\mathrm{NaHCO}_{3}$, vasopressors, intubation, IV fluids \\
\hline $49 \mathrm{M}$ & Ethanol & $\begin{array}{l}\text { RD, CNS, HGY, MA, AG, OG, } \\
\text { PNC, GIB, HYS, CPT, PLT, } \\
\text { PCT, WBC }\end{array}$ & Yes & No & $\begin{array}{l}\text { Fomepizole, vasopressors, benzodiazepines, glucose, opioids, } \\
\text { continuous renal replacement, intubation, IV fluids, transfusion }\end{array}$ \\
\hline $32 \mathrm{M}$ & Ethanol & $\mathrm{CNS}$ & No & No & Folate, thiamine, IV fluids \\
\hline $30 \mathrm{M}$ & Ethanol & $\mathrm{RD}, \mathrm{CNS}$ & Unknown & Unknown & Intubation, IV fluids \\
\hline $31 \mathrm{~F}$ & Ethanol & $\mathrm{CNS}$ & Unknown & Unknown & None listed \\
\hline $60 \mathrm{M}$ & Ethanol & $\begin{array}{l}\mathrm{HT}, \mathrm{AP}, \mathrm{CNS}, \mathrm{HCN}, \mathrm{MA}, \mathrm{AG}, \\
\text { PNC, CPT }\end{array}$ & Yes & Unknown & $\begin{array}{l}\text { Vasopressors, antiarrhythmics, antihypertensives, antipsychotics, } \\
\text { benzodiazepines, opioids, intubation, IV fluids }\end{array}$ \\
\hline $48 \mathrm{~F}$ & Heroin & HT, VD, CNS, MA, HPT, AKI & Yes & No & NAC, vasopressors, CPR, intubation, IV fluids \\
\hline $25 \mathrm{M}$ & Heroin & $\mathrm{HT}, \mathrm{AP}, \mathrm{RD}, \mathrm{CNS}$ & Yes & Yes & Naloxone, vasopressors, benzodiazepines, intubation \\
\hline $32 \mathrm{M}$ & Heroin & HT, RD, CNS & Unknown & Unknown & Naloxone, vasopressors, CPR, intubation, IV fluids \\
\hline $35 \mathrm{M}$ & Heroin & None listed & No & No & Opioids \\
\hline $66 \mathrm{M}$ & Metaxalone & HT, CNS, DLM, RFX, AKI & Yes & Yes & Benzodiazepines, intubation, IV fluids \\
\hline $50 \mathrm{M}$ & Metformin & $\mathrm{HT}, \mathrm{RD}, \mathrm{CNS}, \mathrm{MA}, \mathrm{AKI}$ & Yes & Yes & Vasopressors, continuous renal replacement, intubation, IV fluids \\
\hline $67 \mathrm{M}$ & Metformin & $\begin{array}{l}\text { HT, QTC, ALI, RD, CNS, MA, } \\
\text { AG, GIB, CPT, WBC, AKI }\end{array}$ & Yes & Unknown & $\begin{array}{l}\text { Calcium, methylene blue, } \mathrm{NaHCO}_{3} \text {, thiamine, vasopressors, } \\
\text { benzodiazepines, neuromuscular blockers, opioids, } \\
\text { corticosteroids, continuous renal replacement, intubation, IV } \\
\text { fluids }\end{array}$ \\
\hline$>89 \mathrm{~F}$ & Metformin & HT, QTC, CNS, MA, AG, AKI & Unknown & Unknown & IV fluids \\
\hline $57 \mathrm{M}$ & Methamphetamine & HT, TC, MI, RD, CNS, MA, AG & Yes & No & None listed \\
\hline
\end{tabular}


Table 34 (continued)

\begin{tabular}{|c|c|c|c|c|c|}
\hline $\begin{array}{l}\text { Age/ } \\
\text { gender }^{\mathrm{a}}\end{array}$ & Agents involved & Clinical findings & $\begin{array}{l}\text { Life } \\
\text { support } \\
\text { withdrawn }\end{array}$ & $\begin{array}{l}\text { Brain } \\
\text { death } \\
\text { confirmed }\end{array}$ & Treatment $^{\mathrm{b}}$ \\
\hline $35 \mathrm{M}$ & Methamphetamine & $\begin{array}{l}\text { HT, TC, QRS, MI, SZ, MA, HPT, } \\
\text { CPT, AKI, RBM }\end{array}$ & Yes & No & Vasopressors, benzodiazepines, intubation, IV fluids \\
\hline $55 \mathrm{M}$ & Methanol & SZ, MA, AG, OG & Yes & Unknown & $\begin{array}{l}\text { Fomepizole, antiarrhythmics, benzodiazepines, hemodialysis, } \\
\text { intubation, IV fluids }\end{array}$ \\
\hline $84 \mathrm{~F}$ & $\begin{array}{l}\text { Methylene } \\
\text { chloride }\end{array}$ & None listed & No & No & None listed \\
\hline $29 \mathrm{M}$ & Methylfentanyl & $\begin{array}{l}\text { HT, TC, HYT, MI, RD, CNS, MA, } \\
\text { HPT, CPT, AKI, RBM }\end{array}$ & Yes & No & $\begin{array}{l}\text { Naloxone, vasopressors, benzodiazepines, opioids, intubation, IV } \\
\text { fluids }\end{array}$ \\
\hline $16 \mathrm{M}$ & $\begin{array}{l}\text { Opioid } \\
\text { unspecified }\end{array}$ & $\begin{array}{l}\text { HTN, HT, TC, ALI, CNS, MA, } \\
\text { HPT }\end{array}$ & Yes & No & $\begin{array}{l}\text { Atropine, naloxone, } \mathrm{NaHCO}_{3} \text {, vasopressors, antihypertensives, } \\
\text { benzodiazepines, opioids, CPR, intubation }\end{array}$ \\
\hline $54 \mathrm{M}$ & Quetiapine & QTC, RD, CNS & No & No & IV fluids \\
\hline $71 \mathrm{M}$ & Rasburicase & $\begin{array}{l}\text { HT, ALI, CNS, MA, MET, HYS, } \\
\text { AKI }\end{array}$ & No & No & $\begin{array}{l}\text { Methylene blue, vasopressors, glucose, continuous renal } \\
\text { replacement, intubation, IV fluids, transfusion }\end{array}$ \\
\hline $65 \mathrm{~F}$ & Sodium hydroxide & RD, CNS, CRV, INT, OTH2 & Yes & Yes & Vasopressors, opioids, intubation, IV fluids \\
\hline $58 \mathrm{~F}$ & Verapamil & $\begin{array}{l}\mathrm{HT}, \mathrm{BC}, \mathrm{AVB}, \mathrm{AP}, \mathrm{RD}, \mathrm{CNS}, \mathrm{MA}, \\
\text { PNC, AKI }\end{array}$ & No & No & None listed \\
\hline $17 \mathrm{M}$ & Vitamin A & $\begin{array}{l}\text { HT, TC, VD, RD, CNS, MA, } \\
\text { HYS, PLT, WBC }\end{array}$ & Yes & Unknown & $\begin{array}{l}\text { Calcium, insulin-euglycemic therapy, lipid resuscitation, } \\
\text { vasopressors, benzodiazepines, CPR, ECMO, intubation }\end{array}$ \\
\hline
\end{tabular}

Fatalities reported with known toxicological exposure: based on response from medical toxicologist "Did the patient have a toxicological exposure?" equals yes with known agent(s)

$w k$ weeks, $m$ months, $A G$ anion gap, $A G T$ agitation, $A K I$ Acute kidney injury, $A L I$ acute lung injury/ARDS, $A P$ aspiration pneumonia, $A V B$ AV block, $B C$ bradycardia, $B P$ bradypnea, $C N S$ coma/CNS depression, $C P T$ coagulopathy, $C R V$ corrosive injury, $D L M$ delirium, EPS dystonia/rigidity, GIB GI bleeding, HCN hallucinations, HGY hypoglycemia, HPT hepatoxicity, HT hypotension, HTN hypertension, HYS hemolysis, HYT hyperthermia, INT intestinal ischemia, $M A$ metabolic acidosis, $M E T$ methemoglobinemia, $M I$ myocardial injury/ischemia, NP neuropathy, $O G$ osmole gap, OTH1 rash, OTH2 skin blisters, necrosis, PCT pancytopenia, PLT thrombocytopenia, PNC pancreatitis, PST paresthesia, QRS QRS prolongation, QTc QTc prolongation, $R A D$ asthma/reactive airway disease, $R B M$ rhabdomyolysis, $R D$ respiratory depression, $R F X$ hyperreflexia/tremor, $S Z$ seizures, $T C$ tachycardia, $V D$ ventricular dysrhythmia, $W B C$ leukocytosis, $W K N$ weakness/paralysis, $B A L$ dimercaprol, $C P R$ cardiopulmonary resuscitation, ECMO extracorporeal membrane oxygenation, $\mathrm{NAC}$ n-acetyl cysteine, $\mathrm{NaHCO}_{3}$ sodium bicarbonate

${ }^{a}$ Age in years unless otherwise stated

${ }^{\mathrm{b}}$ Pharmacological and non-pharmacological support as reported by medical toxicologist

number of cases entered is multifactorial, probably a major reason is our continued quality assurance efforts and dropping poorly performing sites from the Consortium.

The Registry serves as a large source of information on poisoning cases as evaluated by bedside medical toxicologists. Although the Toxic Registry is not technically population based, it does have multiple sites broadly around the USA. As such, it may be used in conjunction with other types of registries such as poison centers and health agencies to produce a more detailed understanding of poisoning trends, novel exposures, and their public health implications.

Information on novel exposure surveillance is not included with this annual report, but is being analyzed with results to be reported separately.

Overall, this annual report finds that the most common agent classes, agents, demographics, types of encounters, toxidromes, and treatments remain similar year-to-year. Some notable trends in the Registry as well as trends in a larger national context are discussed.

\section{Use of Extracorporeal Membrane Oxygenation}

The ToxIC case registry has been gathering data on extracorporeal membrane oxygenation (ECMO) since 2013. Analysis of these cases is presented in Table 44. Over 5 years, a total of 62 patients were recorded as receiving ECMO, accounting for a very small percentage of patients in the overall registry. The number of cases per year was relatively consistent, other than in 2015 where there were more cases than typically seen. ECMO patients were relatively younger compared to the total registry sample, with an overall mean age of 22 years. Most of the cases were likely to be related to a toxicologic exposure, with intentional exposures predominating. The most frequently encountered agent classes were analgesics, opioids, cardiovascular agents, psychiatric medications, or a diabetic medication, specifically metformin. Though ECMO is generally considered only in the most critically ill patients, the majority of the patients (76\%) survived. This may be related to the superior prognosis of critically ill poisoned patients as compared to patients who are ill from other medical causes. 
Table 352017 fatalities reported in ToxIC Registry with known toxicological exposure: multiple agents

\begin{tabular}{|c|c|c|c|c|c|}
\hline $\begin{array}{l}\text { Age/ } \\
\text { gender }^{\mathrm{a}}\end{array}$ & Agents involved & Clinical findings & $\begin{array}{l}\text { Life } \\
\text { support } \\
\text { withdrawn }\end{array}$ & $\begin{array}{l}\text { Brain } \\
\text { death } \\
\text { confirmed }\end{array}$ & Treatment $^{\mathrm{b}}$ \\
\hline $52 \mathrm{~F}$ & $\begin{array}{l}\text { Acetaminophen, } \\
\text { hydrocodone }\end{array}$ & None listed & No & No & None listed \\
\hline $86 \mathrm{~F}$ & $\begin{array}{l}\text { Acetaminophen, } \\
\text { ibuprofen }\end{array}$ & $\mathrm{TC}$ & Yes & Unknown & NAC, \\
\hline $60 \mathrm{~F}$ & $\begin{array}{l}\text { Alprazolam, } \\
\text { clonazepam, } \\
\text { quetiapine }\end{array}$ & $\mathrm{RD}, \mathrm{CNS}$ & No & No & Intubation, IV fluids \\
\hline $43 \mathrm{~F}$ & $\begin{array}{l}\text { Alprazolam, } \\
\text { gabapentin }\end{array}$ & QRS, QTC, MI, RD, MA, AG & Yes & Yes & $\begin{array}{l}\mathrm{NaHCO}_{3} \text {, vasopressors, continuous renal } \\
\text { replacement, CPR, intubation, IV fluids }\end{array}$ \\
\hline $35 \mathrm{~F}$ & $\begin{array}{l}\text { Amitriptyline, } \\
\text { buprenorphine, } \\
\text { pregabalin, } \\
\text { lorazepam }\end{array}$ & HT, TC, QRS, RD, CNS, MA, AG & No & No & $\begin{array}{l}\text { Calcium, dantrolene, lipid resuscitation, } \\
\text { naloxone, } \mathrm{NaHCO}_{3} \text {, vasopressors, opioids, } \\
\text { intubation, IV fluids }\end{array}$ \\
\hline $33 \mathrm{M}$ & $\begin{array}{l}\text { Amitriptyline, } \\
\text { clonazepam }\end{array}$ & $\begin{array}{l}\text { TC, QRS, QTC, ALI, AP, RD, CNS, } \\
\text { AG, CPT, RBM }\end{array}$ & No & No & $\mathrm{NaHCO}_{3}$, intubation, IV fluids \\
\hline $29 \mathrm{~F}$ & $\begin{array}{l}\text { Amlodipine, } \\
\text { nifedipine, } \\
\text { metoprolol }\end{array}$ & $\begin{array}{l}\text { HT, BC, VD, RD, CNS, SZ, HGY, MA, } \\
\text { AG, HPT, HYS }\end{array}$ & No & No & $\begin{array}{l}\text { Calcium, glucagon, insulin-euglycemic therapy, } \\
\mathrm{NaHCO}_{3} \text {, vasopressors, benzodiazepines, } \\
\text { glucose, opioids, hemodialysis, CPR, } \\
\text { intubation }\end{array}$ \\
\hline $75 \mathrm{M}$ & $\begin{array}{l}\text { Atenolol, diltiazem, } \\
\text { amiodarone }\end{array}$ & HT, BC, VD, QRS, RD, EPS & Yes & Yes & $\begin{array}{l}\text { Calcium, glucagon, vasopressors, } \\
\text { benzodiazepines, neuromuscular blockers, } \\
\text { continuous renal replacement, intubation, IV } \\
\text { fluids }\end{array}$ \\
\hline $52 \mathrm{~F}$ & $\begin{array}{l}\text { Chlorzoxazone, } \\
\text { amitriptyline, } \\
\text { tramadol }\end{array}$ & CNS, HPT & Unknown & Unknown & NAC \\
\hline $26 \mathrm{~F}$ & $\begin{array}{l}\text { Clonazepam, doxepin, } \\
\text { fluoxetine, } \\
\text { quetiapine, } \\
\text { trazodone, } \\
\text { bupropion }\end{array}$ & $\mathrm{HT}, \mathrm{BC}, \mathrm{VD}, \mathrm{RD}, \mathrm{CNS}, \mathrm{MA}$ & No & No & $\begin{array}{l}\text { Naloxone, vasopressors, antiarrhythmics, CPR, } \\
\text { cardioversion, intubation, IV fluids, therapeutic } \\
\text { hypothermia }\end{array}$ \\
\hline $11 \mathrm{~m} \mathrm{M}$ & $\begin{array}{l}\text { Cocaine, } \\
\text { methamphetamine }\end{array}$ & HT, TC, RAD, AGT & No & No & Benzodiazepines \\
\hline $12 \mathrm{~F}$ & Diazepam, gabapentin & HT, TC, BP, HYT, RD, CNS, MA, AG & No & No & $\begin{array}{l}\text { Naloxone, neuromuscular blockers, intubation, } \\
\text { IV fluids }\end{array}$ \\
\hline $39 \mathrm{~F}$ & $\begin{array}{l}\text { Digoxin, diltiazem, } \\
\text { cardiovascular agent } \\
\text { unspecified, } \\
\text { warfarin }\end{array}$ & $\begin{array}{l}\mathrm{HT}, \mathrm{VD}, \mathrm{ALI}, \mathrm{RD}, \mathrm{CNS}, \mathrm{MA}, \mathrm{CPT} \text {, } \\
\text { AKI }\end{array}$ & Yes & Unknown & $\begin{array}{l}\text { Calcium, digoxin Fab, glucagon, } \\
\text { insulin-euglycemic therapy, lipid resuscitation, } \\
\mathrm{NaHCO}_{3} \text {, vasopressors, antiarrhythmics, } \\
\text { glucose, CPR, cardioversion, intubation, IV } \\
\text { fluids, pacemaker }\end{array}$ \\
\hline $41 \mathrm{~F}$ & Ethanol, lorazepam & CNS & Unknown & Unknown & Antipsychotics, benzodiazepines, \\
\hline $30 \mathrm{~F}$ & $\begin{array}{l}\text { Fentanyl, quinine, } \\
\text { buclizine, methyl } \\
\text { norfentanyl }\end{array}$ & BP, MI, AP, CNS, MA, AKI, RBM & Yes & No & Naloxone, opioids, intubation, IV fluids \\
\hline $34 \mathrm{M}$ & $\begin{array}{l}\text { Heroin, cocaine, } \\
\text { levamisole }\end{array}$ & $\mathrm{HT}, \mathrm{BC}, \mathrm{AP}, \mathrm{RD}, \mathrm{WBC}$ & Yes & Yes & CPR, IVF, therapeutic hypothermia \\
\hline $26 \mathrm{~F}$ & Heroin, cocaine & AVB, CNS, AG, HPT, RBM & Yes & Yes & $\mathrm{NaHCO}_{3}$, vasopressors, opioids, intubation \\
\hline $25 \mathrm{M}$ & $\begin{array}{l}\text { Heroin, ethanol, } \\
\text { alprazolam, cocaine }\end{array}$ & $\begin{array}{l}\text { HT, MI, RD, CNS, MA, AG, HPT, } \\
\text { PNC, AKI }\end{array}$ & Yes & Yes & $\begin{array}{l}\text { Vasopressors, CPR, ECMO, intubation, IV fluids, } \\
\text { therapeutic hypothermia }\end{array}$ \\
\hline $20 \mathrm{~F}$ & $\begin{array}{l}\text { Ibuprofen, citalopram, } \\
\text { trazodone, } \\
\text { penicillin, ethanol }\end{array}$ & CNS & No & No & NAC, IVF \\
\hline $56 \mathrm{M}$ & $\begin{array}{l}\text { Imipramine, } \\
\text { cyclobenzaprine, } \\
\text { hydrocodone }\end{array}$ & QTC, RD, CNS & No & No & Benzodiazepines, intubation, IV fluids \\
\hline $52 \mathrm{M}$ & $\begin{array}{l}\text { Methamphetamine, } \\
\text { amphetamine }\end{array}$ & $\begin{array}{l}\text { HT, MI, AP, RD, AGT, CNS, MA, HPT, } \\
\text { WBC, AKI, RBM }\end{array}$ & Yes & Unknown & None listed \\
\hline $31 \mathrm{M}$ & & None listed & Yes & No & None listed \\
\hline
\end{tabular}


Table 35 (continued)

\begin{tabular}{|c|c|c|c|c|c|}
\hline $\begin{array}{l}\text { Age/ } \\
\text { gender }^{\mathrm{a}}\end{array}$ & Agents involved & Clinical findings & $\begin{array}{l}\text { Life } \\
\text { support } \\
\text { withdrawn }\end{array}$ & $\begin{array}{l}\text { Brain } \\
\text { death } \\
\text { confirmed }\end{array}$ & Treatment $^{\mathrm{b}}$ \\
\hline & $\begin{array}{l}\text { MDMA, } \\
\text { hydromorphone, } \\
\text { methadone, } \\
\text { marijuana }\end{array}$ & & & & \\
\hline $57 \mathrm{M}$ & $\begin{array}{l}\text { Nortriptyline, } \\
\text { gabapentin }\end{array}$ & QRS, MI, RD, CNS & No & No & $\mathrm{NaHCO}_{3}$, intubation, IV fluids \\
\hline $52 \mathrm{M}$ & $\begin{array}{l}\text { Olanzapine, } \\
\text { hydrochlorothiazide }\end{array}$ & TC, DLM & No & No & Benzodiazepines, IV fluids \\
\hline $14 \mathrm{~F}$ & Propofol, ketamine & HT, BC, MI, RD, SZ, MA, AG & Yes & Unknown & Vasopressors, hemodialysis, ECMO, intubation \\
\hline $63 \mathrm{M}$ & $\begin{array}{l}\text { Propranolol, } \\
\text { citalopram }\end{array}$ & $\mathrm{HT}, \mathrm{BC}, \mathrm{AVB}, \mathrm{RD}, \mathrm{CNS}$ & No & No & $\begin{array}{l}\text { Glucagon, vasopressors, antiarrhythmics, } \\
\text { benzodiazepines, neuromuscular blockers, } \\
\text { CPR, intubation, IV fluids }\end{array}$ \\
\hline $33 \mathrm{~F}$ & $\begin{array}{l}\text { Propranolol, } \\
\text { escitalopram, } \\
\text { lamotrigine }\end{array}$ & $\begin{array}{l}\text { HT, TC, BC, HYT, VD, QRS, QTC, } \\
\text { AVB, MI, RD, AGT, CNS, DLM, } \\
\text { EPS, RFX, SZ, HGY, MA, AG, PLT, } \\
\text { RBM }\end{array}$ & Yes & No & $\begin{array}{l}\text { Anticoagulation reversal, calcium, facto } \\
\text { replacement, glucagon, insulin-euglycemic } \\
\text { therapy, } \mathrm{NaHCO}_{3} \text {, vasopressors, } \\
\text { antiarrhythmics, benzodiazepines, glucose, } \\
\text { intubation, IV fluids, transfusion }\end{array}$ \\
\hline $13 \mathrm{~F}$ & $\begin{array}{l}\text { Quetiapine, } \\
\text { acetaminophen }\end{array}$ & HT, ALI, CNS, MA, AG, AKI & Yes & Yes & NAC, hemodialysis, ECMO, intubation, IV fluids \\
\hline $47 \mathrm{~F}$ & $\begin{array}{l}\text { Trazodone, ethanol, } \\
\text { heroin }\end{array}$ & QTC, RFX, GIB & Yes & Unknown & CPR, intubation, IV fluids, transfusion \\
\hline $39 \mathrm{~F}$ & $\begin{array}{l}\text { U47700 (designer } \\
\text { opioid), venlafaxine, } \\
\text { gabapentin, doxepin }\end{array}$ & BP, ALI, AP, RD, CNS, MA & Yes & Yes & IV fluids \\
\hline
\end{tabular}

Fatalities reported with known toxicological exposure: based on response from medical toxicologist "Did the patient have a toxicological exposure?" equals yes with known agent(s)

$w k$ weeks, $m$ months, $A G$ anion gap, $A G T$ agitation, $A K I$ Acute kidney injury, $A L I$ acute lung injury/ARDS, $A P$ aspiration pneumonia, $A V B$ AV block, $B C$ bradycardia, $B P$ bradypnea, $C N S$ coma/CNS depression, $C P T$ coagulopathy, $C R V$ corrosive injury, $D L M$ delirium, EPS dystonia/rigidity, GIB GI bleeding, HCN hallucinations, HGY hypoglycemia, HPT hepatoxicity, HT hypotension, HTN hypertension, HYS hemolysis, HYT hyperthermia, INT intestinal ischemia, MA metabolic acidosis, MET methemoglobinemia, $M I$ myocardial injury/ischemia, NP neuropathy, OG osmole gap, OTHI rash, OTH2 skin blisters, necrosis, PCT pancytopenia, PLT thrombocytopenia, PNC pancreatitis, PST paresthesia, QRS QRS prolongation, QTc QTc prolongation, $R A D$ asthma/reactive airway disease, $R B M$ rhabdomyolysis, $R D$ respiratory depression, $R F X$ hyperreflexia/tremor, $S Z$ seizures, $T C$ tachycardia, $V D$ ventricular dysrhythmia, $W B C$ leukocytosis, $W K N$ weakness/paralysis, $B A L$ dimercaprol, $C P R$ cardiopulmonary resuscitation, ECMO extracorporeal membrane oxygenation, $\mathrm{NAC}$ n-acetyl cysteine, $\mathrm{NaHCO}_{3}$ sodium bicarbonate

${ }^{a}$ Age in years unless otherwise stated

${ }^{\mathrm{b}}$ Pharmacological and non-pharmacological support as reported by medical toxicologist

Table 36 Most common drugs associated with ADRs

\begin{tabular}{ll}
\hline & $N(\%)^{\mathrm{a}}$ \\
\hline Lithium & $14(4.1)$ \\
Valproic acid & $13(3.8)$ \\
Haloperidol & $10(2.9)$ \\
Bupropion & $9(2.7)$ \\
Phenytoin & $9(2.7)$ \\
Clonazepam & $8(2.4)$ \\
Diphenhydramine & $8(2.4)$ \\
Baclofen & $7(2.1)$ \\
\hline
\end{tabular}

ADRs adverse drug reactions

${ }^{a}$ Percentages are calculated out of the total number of cases reporting an ADR $(N=339)$
Alternatively, there may be an inconsistent reporting of deaths in the Registry which could have increased this figure. Since a patient might die at some time after a toxicology consult and the Registry case entry, the consultant would need to actively go back and update the case after a death occurs.

\section{Exposures in Pediatric Patients}

As with the Registry overall, analgesics and antidepressants were the most commonly reported agent classes in pediatric patients (Table 8 ). The anticholinergic/antihistamine class was the next most common in pediatrics, though it was sixth overall in the Registry. The majority of pediatric patients with anticholinergic/antihistamine exposures were in the 1318 year old age group. Opioids were ranked sixth in agent class frequency for pediatrics with the majority of exposures 
Table 37 Antidotal therapy

\begin{tabular}{ll}
\hline & $N(\%)^{\mathrm{a}}$ \\
\hline$N$-acetylcysteine & $820(28.3)$ \\
Naloxone/nalmefene & $633(21.9)$ \\
Sodium bicarbonate & $343(11.8)$ \\
Thiamine & $267(9.2)$ \\
Folate & $157(5.4)$ \\
Fomepizole & $102(3.5)$ \\
Calcium & $74(2.6)$ \\
Physostigmine & $66(2.3)$ \\
Glucagon & $63(2.2)$ \\
Octreotide & $57(2.0)$ \\
Cyproheptadine & $36(1.2)$ \\
L-carnitine & $34(1.2)$ \\
Vitamin K & $34(1.2)$ \\
Atropine & $33(1.1)$ \\
Insulin-euglycemic therapy & $32(1.1)$ \\
Flumazenil & $31(1.1)$ \\
Lipid resuscitation & $27(0.9)$ \\
Pyridoxine & $24(0.8)$ \\
Fab for digoxin & $16(0.6)$ \\
Methylene blue & $11(0.4)$ \\
Dantrolene & $8(0.3)$ \\
Hydroxocobalamin & $8(0.3)$ \\
Bromocriptine & $6(0.2)$ \\
2-PAM & $4(0.2)$ \\
Coagulation factor replacement & $3(0.1)$ \\
Silimarin & $2(0.1)$ \\
Anticoagulation reversal & $2(0.1)$ \\
Nitrites & $1(<0.1)$ \\
Total & $1(<0.1)$ \\
& $2895(100)$ \\
\hline
\end{tabular}

${ }^{\text {a }}$ Percentages are out of the total number of antidotes administered (2895); 2319 registry cases $(30.6 \%)$ received at least one antidote. Cases may have involved the use of multiple antidotes

involving 13-18 year olds, though they were also the most common agent class in those under age 2 .

The most commonly reported pediatric agent classes in ToxIC are notable in that they are dissimilar to those reported by the National Poison Data System (NPDS) [9]. The NPDS describes the most common agent class exposures reported in children less than or equal to 5 years old. When the same subset of patients in the ToxIC database is compared, there are differences in the frequency of agent classes reported. Whereas the NPDS reported the most common pediatric exposures as involving cosmetic and household cleaning products, these were infrequently reported in the ToxIC database. Household products were ranked 15th in frequency in ToxIC, making up just $2.5 \%$ of pediatric exposures. The other nonpharmaceutical class made up only $0.3 \%$ of pediatric
Table 38 Antivenom therapy

\begin{tabular}{ll}
\hline & $N(\%)^{\mathrm{a}}$ \\
\hline Crotalidae polyvalent immune fab (ovine) & $173(92.0)$ \\
Other snake antivenom & $7(3.7)$ \\
Scorpion antivenom & $3(1.6)$ \\
Spider antivenom & $2(1.1)$ \\
Not recorded & $3(1.6)$ \\
Total & $188(100)$ \\
\hline
\end{tabular}

${ }^{\text {a }}$ Percentages are out of the total number of antivenom treatments administered $(N=188)$

exposures. The leading agent class in children $\leq 5$ years old in the ToxIC database was cardiovascular agents (15.6\%), though this agent class made up only $2.1 \%$ of the exposures in the NPDS [9]. Opioids (8.4\%) and sympathomimetics $(7.7 \%)$ were the next most frequently reported agents classes in the ToxIC database. The NPDS reported foreign bodies/ toys/miscellaneous as the fourth most common agent class (6.5\%), compared to being one of the least commonly reported agent classes in ToxIC $(0.3 \%)$.

Of note, the most recent NPDS data available are from 2016, though the agent classes reported are consistent with prior NPDS reports and would not be expected to significantly change in 2017 [10, 11]. Additionally, there are some differences in the categorization of agents into agent classes between the NPDS and ToxIC, though the general trends are still clear. These differences in agent class reporting between the NPDS and ToxIC support the idea that the ToxIC database represents a unique population of patients, with an inclination

Table 39 Supportive care- pharmacological

\begin{tabular}{ll}
\hline & $N(\%)^{\mathrm{a}}$ \\
\hline Benzodiazepines & $1756(49.1)$ \\
Opioids & $511(14.3)$ \\
Vasopressors & $302(8.4)$ \\
Antipsychotics & $250(7.0)$ \\
Neuromuscular blockers & $197(5.5)$ \\
Anticonvulsants & $146(4.1)$ \\
Glucose (concentration $>5 \%)$ & $137(3.8)$ \\
Antihypertensives & $83(2.3)$ \\
Albuterol (or other bronchodilator) & $62(1.7)$ \\
Corticosteroids & $56(1.6)$ \\
Antiarrhythmics & $41(1.1)$ \\
Beta blockers & $24(0.7)$ \\
Vasodilators & $9(0.3)$ \\
Total & $3574(100)$
\end{tabular}

${ }^{a}$ Percentages are out of the total number of treatments administered (3574); 2501 registry cases (33.0\%) received at least one form of pharmacological treatment. Cases may have involved the use of multiple forms of treatment 
Table 40 Supportive care-non-pharmacological

\begin{tabular}{ll}
\hline & $N(\%)^{\mathrm{a}}$ \\
\hline IV fluid resuscitation & $2912(72.3)$ \\
Intubation/ventilatory management & $919(22.9)$ \\
CPR & $74(1.8)$ \\
Transfusion & $36(0.9)$ \\
Therapeutic hypothermia & $18(0.4)$ \\
Hyperbaric oxygen & $15(0.4)$ \\
ECMO & $14(0.3)$ \\
Cardioversion & $11(0.3)$ \\
Pacemaker & $9(0.2)$ \\
Organ transplantation & $3(0.1)$ \\
Aortic balloon pump & $1(0.02)$ \\
Total & $4012(100)$
\end{tabular}

${ }^{\text {a }}$ Percentages are out of the total number of treatments administered (4012); 3141 registry cases $(41.5 \%)$ received at least one form of nonpharmacological treatment. Cases may have involved the use of multiple forms of treatment. CPR Cardiopulmonary resuscitation, ECMO extracorporeal membrane oxygenation

towards more severe exposures. This is likely due to that fact that the cases entered are ones in which a patient presented for care and were evaluated by a medical toxicologist. Additionally, the differences between agent class frequencies in the two databases may speak to the important role of poison control centers in reducing the overuse of healthcare settings for non-life threatening exposures that can be safely managed at home.

\section{Pediatric Exposures to Drugs of Abuse}

A total of 832 patients from the 2017 ToxIC registry were exposed to drugs of abuse with 123 patients of those being between the ages of $0-18(14.8 \%)$. Pediatric patients exposed to drugs of abuse made up $1.6 \%$ of the total Registry. Cases were included after a manual search of pediatric patients for drugs of abuse. Any additional agents in a polypharmacy exposure were also included. The following agents were

Table 41 Chelation therapy

\begin{tabular}{lr}
\hline & $N(\%)^{\mathrm{a}}$ \\
\hline DMSA & $12(38.7)$ \\
Deferoxamine & $8(25.8)$ \\
Dimercaprol & $6(19.4)$ \\
EDTA & $5(16.1)$ \\
Total & $31(100)$ \\
\hline
\end{tabular}

${ }^{a}$ Percentages are out of the total number of chelation treatments administered (31); 25 registry cases $(0.3 \%)$ received at least one form of chelation treatment. DMSA dimercaptosuccinic acid, EDTA ethylenediaminetetraacetic acid
Table 42 Decontamination

\begin{tabular}{ll}
\hline & $N(\%)^{\mathrm{a}}$ \\
\hline Activated charcoal & $226(79.6)$ \\
External irrigation & $23(8.1)$ \\
Whole bowel irrigation & $22(7.7)$ \\
Gastric lavage & $13(4.6)$ \\
Total & $284(100)$ \\
\hline
\end{tabular}

${ }^{\text {a }}$ Percentages are out of the total number of treatments administered (334); 264 registry cases $(3.5 \%)$ received at least one form of decontamination

included: 25I-NBOMe, alprazolam, bupropion, butanoyl-4fluorofentanyl, caffeine, cannabinoid non-synthetic, cannabinoid synthetic, clonidine, cocaine, delta-9-tetrahydrocannabinol, diphenhydramine, ethanol, fentanyl, fluoxetine, gamma hydroxybutyrate, heroin, hydroxyzine, ketamine, LSD (lysergic acid diethylamide), marijuana, methamphetamine, methylenedioxymethamphetamine, methylnorfentanyl, methylphenidate, molly, N-allyl norfentanyl, nicotine, oxycodone, paliperidone, pharmaceutical THC, phencyclidine, phenylephrine, psychoactive unspecified, quetiapine, sympathomimetic unspecified, tramadol, trazodone, and valproic acid. Those patients presenting for withdrawal were excluded from the data analysis. The majority of the pediatric patients were between the ages of $13-18$ (54\%) followed by ages 2-6 $(22 \%)$. Fifty-nine percent identified as Caucasian and nonHispanic (41\%). Pediatric patients were more likely to present due to an intentional exposure of a non-pharmaceutical agent (38.2\%). The most commonly reported class of drug was psychoactives $(46.3 \%)$ followed by sympathomimetics $(43.9 \%)$. The majority of patients had a single agent exposure (61.8\%) as opposed their adult counterparts. There were 2 pediatric deaths related to drugs of abuse. The first was an 11-monthold Caucasian male admitted after an unintentional exposure to cocaine and methamphetamine. The second was a 14-yearold female who received ketamine and propofol. She later developed propofol infusion syndrome and sustained cardiac

Table 43 Enhanced elimination

\begin{tabular}{ll}
\hline & $N(\%)^{\mathrm{a}}$ \\
\hline Urinary alkalinization & $64(26.4)$ \\
Hemodialysis (toxin removal) & $60(24.8)$ \\
Continuous renal replacement therapy & $60(24.8)$ \\
Hemodialysis (other indication) & $45(18.6)$ \\
Multiple-dose activation charcoal & $11(4.5)$ \\
Exchange transfusion & $2(0.8)$ \\
Total & $242(100)$ \\
\hline
\end{tabular}

${ }^{\text {a }}$ Percentages are out of the total number of treatments administered (242); 226 registry cases $(3.0 \%)$ received at least one form of enhanced elimination 
arrest. The circumstances surrounding ketamine exposure were not described. When analyzed by gender, there were no significant differences in the reason for exposure, agent used or demographics in the pediatric drug of abuse population.

\section{Cannabis-the Trouble with Edibles}

Cannabis is the most commonly used illicit drug in the USA, according to the 2016 National Survey on Drug Use and Health [12]. While cannabis is typically consumed through smoke inhalation, the increase in the legalization of cannabis has made "edibles" (products containing cannabis intended for oral consumption) more accessible as the cannabis market continues to evolve [13]. There is often significant variation in the potency of "edibles," which can contribute to accidental overdose, especially with inexperienced users. Perhaps more troubling is the unintentional ingestion of edibles by young children and adolescents that can result in significant toxicity, including agitation, ataxia, hallucinations, lethargy, myoclonus, respiratory depression, seizures, and tachycardia [14-16].

Since the first edibles cases were reported in the ToxIC Registry in 2012, there has been a substantial increase in the number of cases involving children $0-6$ years of age [4]. In 2012, there were no "edibles" cases reported to the ToxIC Registry that involved children 0-6 years of age, compared with 10 cases in 2016 (52.6\% of total "edibles" cases) and 8 cases in 2017 (50.0\% of total "edibles" cases) [4, 8]. In 2017, among the children $0-6$ years of age that had unintentionally consumed a product containing cannabis, CNS depression was reported in five, delirium in three, agitation in one, hallucinations in one, respiratory depression in one, and seizures in one, with no fatalities reported. Certainly, this is a concerning trend, and clinicians should be proactive in educating parents about the dangers that "edibles" pose to young children.

\section{Increase in ToxIC Gabapentinoid (GP) Cases}

Gabapentin and pregabalin are substituted derivatives of the neurotransmitter gamma-aminobutyric acid (GABA) labeled in the USA for the treatment of neurologic pain and seizures $[17,18]$. Their weak GABA-mimetic features include feelings of relaxation and euphoria reported by some patients and recreational users [17]. As with the benzodiazepines and other addictive GABA-mimetics, withdrawal symptoms can occur and tolerance to the euphoria develops, increasing their addiction potential [17]. This, combined with a global increase in GP prescribing for a variety of on- and off-label uses (e.g., anxiety, insomnia, and recreational drug withdrawal, among others), is thought to help explain the recent spikes in the numbers of GP abuse cases reported to pharmacovigilance databases in Europe $[19,20]$. In the USA, although population-level estimates of GP misuse/abuse are not yet 
available, GP misuse/abuse appears on the rise as well [21]. A recent analysis of data from the Researched Abuse, Diversion and Addiction-Related Surveillance (RADARS®) system, a national prescription drug surveillance system covering all states except Hawaii, reported 407 cases of gabapentin diversion in 41 states from 2002 to 2015 and showed steady annual increases of gabapentin diversion from 2002 to 2015, with a 2015 diversion rate comparable to OxyContin [21]. In 2016, the US National Poison Data System (NPDS) reported 3443 intentional (including misuse/abuse, suicide, and unknown) single agent gabapentin exposures, up from 0 in 2011, the first year it was reported separately $[9,22]$. These recent spikes in GP misuse/abuse are mirrored in the numbers of GP cases recorded in ToxIC, up from 54 (1.4\% of all ToxIC cases) in 2010 to 201 (2.7\% of all ToxIC cases) in 2017. Approximately half the ToxIC 2017 cases were suicide attempts and onequarter were misuse/abuse cases.

Some speculate that off-label prescriptions are increasing as physicians attempt to provide non-opioid pain relief to their patients, despite the lack of efficacy and long-term safety data for common off-label pain indications [23, 24]. There are also published case reports of GP abuse by patients with substance use disorders (SUDs), especially prescription opioid misusers and heroin users, and of patients attempting to potentiate the effects of methadone or buprenorphine or to get high from the buprenorphine/gabapentin combination [9, 24, 25]. A recent systematic review concluded that those most at risk of GP addiction were patients with current or past SUDs, particularly opioid and multi-drug users, and that the risk of overdose lethality increases with multiple agent exposures, particularly those involving opioids and sedatives [17]. Potential mechanisms include pharmacodynamic (i.e., additive respiratory depression of opioids and gabapentin) and pharmacokinetic (i.e., increased gabapentin absorption in the upper small intestine after slowed motility from opioid ingestion) factors [26]. In the GP misuse/abuse cases in ToxIC, coma/CNS (central nervous system) depression affects approximately half or more of both the single and multiple agent cases, regardless of coingestant category, and respiratory depression is seen in approximately one-third or more of the multiple agent cases. Overall, the ToxIC data illustrate the range of potentially severe medical outcomes associated with GP misuse/abuse and provide complimentary insight on its burden on US healthcare facilities.

\section{Limitations}

The ToxIC Registry is a unique database that is able to provide an informed reported relationship between exposures and clinical outcomes due to its involvement of actively practicing medical toxicologists. There are, however, some limitations within the Registry. One of these is a possible bias towards more severe case presentations since cases are only entered if they are evaluated at the bedside by a medical toxicologist. Cases in which the patient did not present for care or in which a medical toxicology consult was not called would not have been reported. Therefore, the Registry likely represents a different population from other agencies such as Poison Control Centers. There may also be a bias in the types of cases reported based on regional variations in drug use and abuse and toxicological exposures. The scope of the ToxIC Registry includes medical toxicologists from multiple, diverse locations, but the entire country is not fully or equally represented. Larger, academic medical centers may be more likely to have practicing medical toxicologists and be more likely to be members of the Consortium. Therefore, some areas of the USA and more rural populations may be underrepresented.

Additionally, there may be a reporting bias towards more complicated or more interesting cases at the level of the individuals reporting cases to the Registry. We attempt to limit this as much as possible by way of a written agreement with all participating sites requiring that all cases that are seen be entered into the Registry. Data regarding substances of exposure, or species of envenomation, relies heavily on patient selfreport which is limited by willingness to disclose as well as lack of knowledge about substances involved. This may be particularly true of illicit drugs, making the subset of patients with analytical confirmation of fentanyl analogue exposure particularly valuable. Lastly, improving data quality remains an issue for the registry. While member sites are instructed to complete all applicable data fields in each case, there are still a number of cases with likely incomplete data. This is best demonstrated the fatality cases where no notable signs/symptoms or treatments were reported. Efforts remain underway to improve reporting of data, in particular demographic and fatality data.

\section{Conclusions}

The ToxIC Registry continues to be the only database of its kind, poised to collect data on toxicological exposures that have each been evaluated by a bedside medical toxicologist. While limited to the scope of those participating medical toxicologists, this also enhances the ability of the Registry to correlate exposures to clinical findings. Quality improvement efforts have improved the consistent reporting of information, and these efforts continue to be a major focus of the Registry going forward. The most commonly reported exposures, types of encounters, toxidromes, and clinical signs and symptoms overall remain consistent and represent the current practice of participating medical toxicologists. While nearly threequarters of cases required treatment intervention, fatalities were uncommon.

Acknowledgments Toxicology Investigators Consortium (ToxIC) Study Group Collaborators: 
Algren DA, Alwasiyah D, Beauchamp GA, Bentur Y, Berman AJ, Beuhler MC, Biary R, Bonney C, Boyle KL, Bruccoleri RC, Burns MM, Cahana A, Cannon RD, Caravati EM, Carey J, Chiba T, Christian M, Cook MD, Cumpston K, Davis A, Dribben W, Eisenga BH, Eldos Y, Falkowitz DM, Farkas A, Finkelstein Y, Fisher E, Froberg BA, Ganetsky M, Garlich F, Geib AJ, Gittinger M, Gorodetsky R, Greene SC, Hart K, Hendrickson RG, Hernandez S, Hodgman M, Hoyte C, Judge BS, Kao L, Katz KD, Kessler BD, King JD, Kirschner RI, Kostic M, Leikin JB, Levine M, Lowry JA, Lurie Y, Majlesi N, Malashock H, Manini AF, Marino R, McKay CA Jr., McKeever R, McKeown N, Meadors K, Moore E, Morgan B, Mullins ME, Nacca NE, Niruntarai S, Nogar JN, Olmedo R, Othong R, Riley BD, Rusyniak DE, Schimmel J, Schult R, Schwarz ES, Scoccimarro A, Seifert SA, Shafer S, Smith S, Smolinske SC, Spyres M, Steck A, Stripp M, Sullivan R, Theobald J, Troendle M, Vearrier, Warrick BJ, Watts D, Wills B, Zosel AE.

We also wish to thank study coordinators AB Adefeso, D Hopkins, Julie Licata, Tammy Phan, Andrea Ramirez, Mellissa VandenBerg, and Love Wilson.

Funding Sources This study received funding from the NIH National Institute on Drug Abuse, 1R56DA38366 and 1R01DA037317-01, a data sharing contract with the US Food and Drug Administration and BTG International Inc. (North America).

\section{Compliance with Ethical Standards}

\section{Conflicts of Interest None}

Previous Presentation of Data None

\section{References}

1. Wax PM, Kleinschmidt KC, Brent J. ACMT ToxIC Case Registry Investigators. The Toxicology Investigators Consortium (ToxIC) Registry. J Med Toxicol. 2011;7(4):259-65.

2. Brent J, Wax PM, Schwartz T, et al. The Toxicology Investigators Consortium Case Registry - the 2010 experience. J Med Toxicol. 2011;7(4):266-76.

3. Wiegand TJ, Wax PM, Schwartz T, et al. The Toxicology Investigators Consortium Case Registry - the 2011 experience. J Med Toxicol. 2012;8(4):360-77.

4. Wiegand T, Wax P, Smith E, Hart K, Brent J. The Toxicology Investigators Consortium Case Registry - the 2012 experience. J Med Toxicol. 2013;9(4):380-404.

5. Rhyee SH, Farrugia L, Wiegand T, et al. The Toxicology Investigators Consortium Case Registry-the 2013 experience. J Med Toxicol. 2014;10(4):342-59.

6. Rhyee SH, Farrugia L, Campleman SL, et al. The Toxicology Investigators Consortium Case Registry - the 2014 experience. J Med Toxicol. 2015;11(4):388-409.

7. Farrugia L, Rhyee SH, Campleman SL, et al. The Toxicology Investigators Consortium Case Registry - the 2015 experience. J Med Toxicol. 2016;12(3):224-47.

8. Farrugia L, Rhyee SH, Calello DP, et al. The Toxicology Investigators Consortium Case Registry - the 2016 experience. J Med Toxicol. 2017;13(3):203-26.

9. Gummin DD, Mowry JB, Spyker DA, Brooks DE, Fraser MO, Banner W. 2016 annual report of the American Association of Poison Control Centers' National Poison Data System (NPDS): 34th annual report. Clin Toxicol (Phila). 2017;55(10):1072-252.
10. Mowry JB, Spyker DA, Brooks DE, Zimmerman A, Schauben JL. 2015 annual report of the American Association of Poison Control Centers' National Poison Data System (NPDS): 33rd annual report. Clin Toxicol (Phila). 2016;54(10):924-1109.

11. Mowry JB, Spyker DA, Brooks DE, McMillan N, Schauben JL. 2014 annual report of the American Association of Poison Control Centers' National Poison Data System (NPDS): 32nd annual report. Clin Toxicol (Phila). 2015;53(10):962-1146.

12. Center for Behavioral Health Statistics and Quality. 2016 national survey on drug use and health: detailed tables. Substance Abuse and Mental Health Services Administration, Rockville, MD 2017.

13. Ouellette L, Cearley M, Judge B, Riley B, Jones J. Cooking with cannabis: the rapid spread of (mis)information on YouTube. Am J Emerg Med 2017.

14. Richards JR, Smith NE, Moulin AK. Unintentional cannabis ingestion in children: a systematic review. J Pediatr. 2017;190:142-52.

15. Vo KT, Horng H, Li K, Ho RY, Wu AHB, Lynch KL, et al. Cannabis intoxication case series: the dangers of edibles containing tetrahydrocannabinol. Ann Emerg Med. 2018;71:306-13.

16. Sierzant C, Judge B. Cooking with cannabis: severe toxicity following the use of cannabutter. Clin Toxicol. 2016;54:708.

17. FDA (U.S. Food and Drug Administration). 2011. Neurontin ${ }^{\circledR}$ (gabapentin) capsules, Neurontin ${ }^{\circledR}$ (gabapentin) tablets, Neurontin ${ }^{\circledR}$ (gabapentin) oral solution. NDA 020235/S-036; NDA 020882/S-022; NDA 021129/S-022 FDA Approved Labeling Text dated 03/01/2011. Available: https://www. accessdata.fda.gov/drugsatfda_docs/label/2011/020235s036, 020882s022,021129s022lbl.pdf; accessed 12 Feb 2018.

18. FDA. 2012. Highlights of prescribing information: Lyrica (pregabalin) capsules, CV Lyrica (pregabalin) oral solution, CV. Available: https://www.accessdata.fda.gov/drugsatfda_docs/label/ 2012/021446s028lbl.pdf; accessed 12 Feb 2018.

19. Chiappini S, Schifano F. A decade of gabapentinoid misuse: an analysis of the European Medicines Agency's 'Suspected Adverse Drug Reactions’ database. CNS Drugs. 2016;30(7):647-54.

20. Office for National Statistics [United Kingdom]. 2017. Death related to drug poisoning in England and Wales from 1993 onwards, by cause of death, sex, age and substances involved in the death. Latest release: Deaths related to drug poisoning in England and Wales: 2016 registrations, Released: 2 Aug 2017. Available: https://www.ons.gov.uk/ peoplepopulationandcommunity/birthsdeathsandmarriages/deaths/ bulletins/deathsrelatedtodrugpoisoninginenglandandwales/ 2016registrations; accessed 12 Feb 2018.

21. Buttram ME, Kurtz SP, Dart RC, Margolin ZR. Law enforcementderived data on gabapentin diversion and misuse, 2002-2015: diversion rates and qualitative research findings. Pharmacoepidemiol Drug Saf. 2017;26(9):1083-6.

22. Bronstein AC, Spyker DA, Cantilena LR Jr, Rumack BH, Dart RC. 2011 annual report of the American Association of Poison Control Centers' National Poison Data System (NPDS): 29th annual report. Clin Toxicol (Phila). 2012;50(10):911-1164.

23. Goodman CW, Brett AS. Gabapentin and Pregabalin for pain - is increased prescribing a cause for concern? N Engl J Med. 2017;377(5):411-4. https://doi.org/10.1056/NEJMp1704633.

24. Johansen ME. Gabapentinoid use in the United States 2002 through 2015. JAMA Intern Med. 2018;178(2):292-4.

25. Smith RV, Lofwall MR, Havens JR. Abuse and diversion of gabapentin among nonmedical prescription opioid users in Appalachian Kentucky. Am J Psychiatry. 2015;172(5):487-8.

26. Gomes T, Juurlink DN, Antoniou T, Mamdani MM, Paterson JM, van den Brink W. Gabapentin, opioids, and the risk of opioidrelated death: a population-based nested case-control study. PLoS Med. 2017;14(10):e1002396. 